\title{
Convergence analysis of trigonometric methods for stiff second-order stochastic differential equations
}

\author{
David Cohen · Magdalena Sigg
}

Received: 10 January 2011 / Revised: 15 August 2011 / Published online: 13 November 2011

(C) Springer-Verlag 2011

\begin{abstract}
We study a class of numerical methods for a system of second-order SDE driven by a linear fast force generating high frequency oscillatory solutions. The proposed schemes permit the use of large step sizes, have uniform global error bounds in the position (i.e. independent of the large frequencies present in the SDE) and offer various additional properties. This new family of numerical integrators for SDE can be viewed as a stochastic generalisation of the trigonometric integrators for highly oscillatory deterministic problems.
\end{abstract}

Mathematics Subject Classification (2000) $\quad 65 \mathrm{C} 20 \cdot 60 \mathrm{H} 10$

\section{Introduction}

In this article we present an error analysis for a family of numerical schemes for the solution of the ( $d$-dimensional) stiff second-order SDE

$$
\ddot{X}_{t}+\frac{1}{\varepsilon^{2}} A X_{t}=g\left(X_{t}\right)+B \dot{W}_{t},
$$

where $\varepsilon \ll 1$ is a small parameter, the nonlinearity $g(x) \in \mathbb{R}^{d}$ is a smooth real function and $W_{t}=\left(W_{1}(t), \ldots, W_{m}(t)\right)^{T}$ is a standard $m$-dimensional Wiener process. We will assume that the matrix $A \in \mathbb{R}^{d \times d}$, which does not depend on $\varepsilon$, is a symmetric positive definite matrix such that the norm of $A^{-1}$ is not small compared to $\varepsilon$, that is, all the

D. Cohen $(\varangle) \cdot$ M. Sigg

Mathematisches Institut, Universität Basel, 4051 Basel, Switzerland

e-mail: David.Cohen@unibas.ch

M. Sigg

e-mail: Magdalena.Sigg@unibas.ch 
eigenvalues of $\frac{1}{\varepsilon^{2}} A$ are away from zero. We will also assume that the norm of the matrix $B \in \mathbb{R}^{d \times m}$ is small compared to the norm of the fast force $F(x)=\frac{1}{\varepsilon^{2}} A x$. At this point, we would like to add, that we can also consider the case, where the matrix $A$ has zero as eigenvalues. For ease of presentation, this will be shown only in the last section of the paper. Here and in the following we will work with the Euclidean norm or with norms induced by the Euclidean norm.

We are interested in numerical methods that can attain good accuracy with step sizes whose product with the large frequencies in (1) need not to be small. So that the error bounds in the position of the methods should be independent of the product of the step size with the frequencies of our problem.

The proposed schemes can be viewed as a stochastic generalisation of the trigonometric methods (or exponential integrators) for highly oscillatory deterministic problems (see [2], [5, Chapter XIII] and references therein) and appeared for the first time in [1] for the numerical discretisation of scalar stochastic oscillators with a high frequency. Since a key building-block in the development of stochastic trigonometric methods is the variation-of-constants formula, we begin by rewriting (1) as a system of first order $\operatorname{SDE}\left(\operatorname{setting} X_{t}^{1}:=X_{t}, X_{t}^{2}:=\dot{X}_{t}\right.$ and $\Omega:=\frac{1}{\varepsilon} A^{1 / 2}$ ):

$$
\left(\begin{array}{l}
d X_{t}^{1} \\
d X_{t}^{2}
\end{array}\right)=\left(\begin{array}{cc}
0 & 1 \\
-\Omega^{2} & 0
\end{array}\right)\left(\begin{array}{l}
X_{t}^{1} \\
X_{t}^{2}
\end{array}\right) d t+\left(\begin{array}{c}
0 \\
g\left(X_{t}^{1}\right)
\end{array}\right) d t+\left(\begin{array}{l}
0 \\
B
\end{array}\right) d W_{t}
$$

The variation-of-constants formula for the above equation will then suggest the construction of the stochastic trigonometric schemes (see Sect. 2 for details on the derivation of the numerical methods). After that, we will present the main theorems on the convergence of the schemes in Sects. 3 and 4. It turns out that the proposed schemes offer additional features similar to the one of the exact solution of (2). This will be studied in more details in Sect. 5. Numerical experiments demonstrating the convergence and the good long-time behaviour of the stochastic trigonometric schemes are presented in the final section.

Let us mention that the use of the variation-of-constants formula to derive efficient numerical schemes for large stiff systems of first order differential equations is not new. In the deterministic setting, one may consult, for example, $[6,13]$ and more recently the review [7] on exponential integrators. Stochastic exponential integrators were also considered for the numerical approximation of parabolic stochastic partial differential equations in $[9,10,14]$ and references therein.

We conclude the introduction by mentioning that there are only few numerical works in the literature to solve stiff systems of the form (1). We are only aware of the work [17]. The results given in this article are, in the spirit, closely related to ours; the techniques and the equation considered here are however different. Let us finally mention, that for the linear scalar case and with a frequency $1 / \varepsilon=1$ (non-stiff problems), we are only aware of the works $[8,16]$ and [18]. For a numerical comparison between these schemes and the stochastic trigonometric methods, we refer to [1]. 


\section{Stochastic trigonometric methods}

In this section we recall the definition of the (stochastic) trigonometric schemes. For details on the derivations we refer to [5, Chapter XIII] for the deterministic case and to [1] for the stochastic scalar case.

As stated in the introduction, the main building-block for the construction of the stochastic trigonometric integrators is the variation-of-constants formula. The exact solution of (2) with initial values $X_{0}^{1}=x_{0}$ and $X_{0}^{2}=y_{0}$ satisfies

$$
\begin{aligned}
\left(\begin{array}{l}
X_{t}^{1} \\
X_{t}^{2}
\end{array}\right)= & \left(\begin{array}{cc}
\cos (t \Omega) & \Omega^{-1} \sin (t \Omega) \\
-\Omega \sin (t \Omega) & \cos (t \Omega)
\end{array}\right)\left(\begin{array}{l}
x_{0} \\
y_{0}
\end{array}\right) \\
& +\int_{0}^{t}\left(\begin{array}{c}
\Omega^{-1} \sin ((t-s) \Omega) \\
\cos ((t-s) \Omega)
\end{array}\right) g\left(X_{s}^{1}\right) d s+\int_{0}^{t}\left(\begin{array}{c}
\Omega^{-1} \sin ((t-s) \Omega) \\
\cos ((t-s) \Omega)
\end{array}\right) B d W_{s} .
\end{aligned}
$$

We will sometimes use the notation $t \operatorname{sinc}(t \Omega)$ for $\Omega^{-1} \sin (t \Omega)$, which is defined for arbitrary matrices $\Omega$.

We now discretise the above deterministic and Ito integrals and thereby obtain a family of explicit trigonometric numerical integrators:

$$
\begin{aligned}
\left(\begin{array}{l}
X_{n+1}^{1} \\
X_{n+1}^{2}
\end{array}\right)= & \left(\begin{array}{cc}
\cos (h \Omega) & \Omega^{-1} \sin (h \Omega) \\
-\Omega \sin (h \Omega) & \cos (h \Omega)
\end{array}\right)\left(\begin{array}{l}
X_{n}^{1} \\
X_{n}^{2}
\end{array}\right) \\
& +\left(\begin{array}{c}
\frac{h^{2}}{2} \Psi g\left(\Phi X_{n}^{1}\right) \\
\frac{h}{2}\left(\Psi_{0} g\left(\Phi X_{n}^{1}\right)+\Psi_{1} g\left(\Phi X_{n+1}^{1}\right)\right)
\end{array}\right)+\left(\begin{array}{c}
\Omega^{-1} \sin (h \Omega) B \Delta W_{n} \\
\cos (h \Omega) B \Delta W_{n}
\end{array}\right),
\end{aligned}
$$

where $h$ denotes the step size of the scheme and $\Delta W_{n}=W\left(t_{n+1}\right)-W\left(t_{n}\right)$ the Wiener increments. Here $\Psi=\psi(h \Omega)$ and $\Phi=\phi(h \Omega)$, where the filter functions $\psi, \phi$ are even, real-valued functions with $\psi(0)=\phi(0)=1$. Moreover, we have $\Psi_{0}=\psi_{0}(h \Omega)$, $\Psi_{1}=\psi_{1}(h \Omega)$ with even functions $\psi_{0}, \psi_{1}$ satisfying $\psi_{0}(0)=\psi_{1}(0)=1$. The purpose of these filter functions is to attenuate numerical resonances, see [5, Chapter XIII] for the deterministic case. The choice of the filter functions may also have a substantial influence on the long-time properties of the method. We will not deal with these issues in the present paper. We note that the family of integrators (3) reduces (in the symmetric case) to the Störmer-Verlet scheme for $\Omega=B=0$ and give the exact solution for $g=0$ and $B=0$.

Example 1 Replacing the deterministic integral in the variation-of-constants formula for the exact solution by its trapezoidal rule approximation yields the following explicit scheme (see [3] for the deterministic case):

$$
\begin{aligned}
\left(\begin{array}{l}
X_{n+1}^{1} \\
X_{n+1}^{2}
\end{array}\right)= & \left(\begin{array}{cc}
\cos (h \Omega) & \Omega^{-1} \sin (h \Omega) \\
-\Omega \sin (h \Omega) & \cos (h \Omega)
\end{array}\right)\left(\begin{array}{l}
X_{n}^{1} \\
X_{n}^{2}
\end{array}\right) \\
& +\frac{h}{2}\left(\begin{array}{c}
\Omega^{-1} \sin (h \Omega) g_{n} \\
g_{n+1}+\cos (h \Omega) g_{n}
\end{array}\right)+\left(\begin{array}{c}
\Omega^{-1} \sin (h \Omega) B \Delta W_{n} \\
\cos (h \Omega) B \Delta W_{n}
\end{array}\right),
\end{aligned}
$$


where $g_{n}=g\left(X_{n}^{1}\right)$. This is exactly method (3) for the choices $\psi(\zeta)=$ $\operatorname{sinc}(\zeta), \psi_{0}(\zeta)=\cos (\zeta), \psi_{1}(\zeta)=1$ and $\phi(\zeta)=1$.

Example 2 Another possibility is to use only the left-hand endpoint to discretise the deterministic integral. This gives us the following filter functions: $\psi(\zeta)=$ $2 \operatorname{sinc}(\zeta), \psi_{0}(\zeta)=2 \cos (\zeta), \psi_{1}(\zeta)=0$ and $\phi(\zeta)=1$. Note that we do not have $\psi_{1}(0)=1$. One recognises the exponential Euler scheme for parabolic problems from [14]. Another discretisation of the deterministic integral (with filter functions $\psi(\zeta)=2(1-\cos (\zeta)) / \zeta^{2}, \psi_{0}(\zeta)=2 \operatorname{sinc}(\zeta), \psi_{1}(\zeta)=0$ and $\left.\phi(\zeta)=1\right)$ gives us the exponentially fitted Euler scheme from [9]. We remark that the stochastic part in the scheme presented in [9] is not treated in the same way as in (3). Moreover, a nice feature of the numerical integrator proposed in [9] is that it takes advantage of a smoothing effect of a linear functional of the noise-term.

Remark 1 Other discretisations of the stochastic integral present in the variationof-constants formula are possible and will lead to various numerical schemes. We can for example use the following approximation:

$$
\int_{0}^{h}\left(\begin{array}{c}
\Omega^{-1} \sin ((h-s) \Omega) \\
\cos ((h-s) \Omega)
\end{array}\right) B d W_{s} \approx\left(\begin{array}{c}
\Omega^{-1} \sin (\tilde{\phi}(h \Omega)) B \Delta W_{0} \\
\cos (\tilde{\phi}(h \Omega)) B \Delta W_{0}
\end{array}\right) .
$$

Taking the trivial choice $\tilde{\phi} \equiv 0$, one obtains the numerical scheme proposed by Tocino in [18] for scalar linear second-order SDE $\ddot{X}_{t}+X_{t}=\alpha \dot{W}_{t}$. However a more natural and appropriate discretisation of the integral is by taking the Riemann left-end points, that is with the choice $\tilde{\phi}(x)=x$, and thus obtain the approximation present in method (3). We will only consider this discretisation in the present paper.

Since we are interested in using large step sizes, we will consider the numerical solution of (1) by method (3) with a step size $h \leq h_{0}$ (with a sufficiently small $h_{0}$ independent of $\varepsilon$ ) for which

$$
\frac{h}{\varepsilon} \geq c_{0}>0
$$

\section{Mean-square convergence analysis}

In this section, we will derive mean-square error bounds over finite time intervals for the family of stochastic trigonometric methods derived in Sect. 2. To do so, we first look at the mean-square error for linear problems, that is equation (1) with $g \equiv 0$. The proofs follow the lines of the scalar case given in [1]. Combining these results with the results from [4], this then permits us to derive order one mean-square error bounds, for the position component, independent of the product of the step size with the large frequencies present in our problem.

Let us first recall the definition of mean-square convergence, see for example [11]. 
Definition 1 A numerical method $\left\{Y_{n}\right\}$ with step size $h$ converges in the mean-square sense with order $\beta>0$ to the solution $Y_{t_{n}}$ of an SDE at time $t_{n}=n h$ if $\beta$ is the largest value such that there exists a positive constant $C$, which does not depend on $h$, and an $h_{0}>0$ such that

$$
\left(\mathbb{E}\left[\left\|Y_{t_{n}}-Y_{n}\right\|^{2}\right]\right)^{1 / 2} \leq C h^{\beta}
$$

for all $h \in\left(0, h_{0}\right)$.

We now show that the local mean-square error in the position of our stochastic trigonometric integrator is of order $\mathcal{O}\left(h^{3 / 2}\right)$ for linear problems.

Lemma 1 Consider the numerical solution of (1) with $g \equiv 0$ by method (3) with a step size $h \leq h_{0}$ (with a sufficiently small $h_{0}$ independent of $\varepsilon$ ) for which $h / \varepsilon \geq c_{0}>0$ holds. The mean-square errors after one step of the numerical scheme satisfy

$$
\begin{aligned}
& \left(\mathbb{E}\left[\left\|X_{1}^{1}-X_{h}^{1}\right\|^{2}\right]\right)^{1 / 2} \leq C \varepsilon h^{1 / 2} \leq C h^{3 / 2} \\
& \left(\mathbb{E}\left[\left\|X_{1}^{2}-X_{h}^{2}\right\|^{2}\right]\right)^{1 / 2} \leq C h^{1 / 2},
\end{aligned}
$$

where the constant $C$ depends on the norm of the matrix $B$, but is independent of $\varepsilon$ and $h$. That is, the local errors are of order (at least) 1/2 uniformly in the frequencies.

Proof Let us start with the local mean-square error in the position. By definition of the method and by the variation-of-constants formula for the exact solution, we obtain

$$
\mathbb{E}\left[\left\|X_{1}^{1}-X_{h}^{1}\right\|^{2}\right]=\mathbb{E}\left[\left\|\int_{0}^{h} \Omega^{-1}(\sin (h \Omega)-\sin ((h-s) \Omega)) B d W_{s}\right\|^{2}\right]
$$

Using the Ito isometry and the triangle inequality, we get

$$
\begin{aligned}
\mathbb{E}\left[\left\|X_{1}^{1}-X_{h}^{1}\right\|^{2}\right] & \leq\|B\|^{2}\left\|\Omega^{-1}\right\|^{2} \int_{0}^{h}\|\sin (h \Omega)-\sin ((h-s) \Omega)\|^{2} d s \\
& \leq C\left\|\Omega^{-1}\right\|^{2} h .
\end{aligned}
$$

Using the definition of the matrix $\Omega$, we end up with the stated bounds for the local error in the position component. The estimate for the error in the velocity component is obtained in a similar way: we have 


$$
\begin{aligned}
\mathbb{E}\left[\left\|X_{1}^{2}-X_{h}^{2}\right\|^{2}\right] & =\mathbb{E}\left[\left\|\int_{0}^{h}(\cos (h \Omega)-\cos ((h-s) \Omega)) B d W_{s}\right\|^{2}\right] \\
& \leq\|B\|^{2} \int_{0}^{h}\|\cos (h \Omega)-\cos ((h-s) \Omega)\|^{2} d s \leq C h .
\end{aligned}
$$

We now turn our attention to the global mean-square error of the stochastic trigonometric integrator (3) for linear systems. We obtain the following result.

Proposition 1 Consider the numerical solution of (1) with $g \equiv 0$ by method (3) with a step size $h \leq h_{0}$ (with a sufficiently small $h_{0}$ independent of $\varepsilon$ ) for which $h / \varepsilon \geq c_{0}>0$ holds. The mean-square errors of the numerical scheme satisfy

$$
\begin{aligned}
& \left(\mathbb{E}\left[\left\|X_{n}^{1}-X_{n h}^{1}\right\|^{2}\right]\right)^{1 / 2} \leq C \varepsilon \leq C h \\
& \left(\mathbb{E}\left[\left\|X_{n}^{2}-X_{n h}^{2}\right\|^{2}\right]\right)^{1 / 2} \leq C T^{1 / 2} \quad \text { for } n h \leq T,
\end{aligned}
$$

where the constant $C$ is independent of $\varepsilon, h$ and $n$ with $n h \leq T$. That is, the global error in the position component is of order 1 uniformly in the frequencies. Unfortunately, we obtain a non-uniform global error in the velocity component.

Remark 2 We would like to point out, that it is not surprising to obtain a non-uniform global error in the velocity in the stochastic case. This was also observed in [17].

Proof We start by substituting the exact solution into the numerical scheme (3) and obtain

$$
\left(\begin{array}{l}
X_{t_{n+1}}^{1} \\
X_{t_{n+1}}^{2}
\end{array}\right)=R \cdot\left(\begin{array}{l}
X_{t_{n}}^{1} \\
X_{t_{n}}^{2}
\end{array}\right)+\left(\begin{array}{c}
\Omega^{-1} \sin (h \Omega) B \Delta W_{n} \\
\cos (h \Omega) B \Delta W_{n}
\end{array}\right)+\left(\begin{array}{l}
d_{n}^{1} \\
d_{n}^{2}
\end{array}\right)
$$

where $t_{n}=n h$, the matrix $R=\left(\begin{array}{cc}\cos (h \Omega) & \Omega^{-1} \sin (h \Omega) \\ -\Omega \sin (h \Omega) & \cos (h \Omega)\end{array}\right)$ and the defects

$$
\begin{aligned}
& d_{n}^{1}=\int_{t_{n}}^{t_{n+1}} \Omega^{-1} \sin \left(\left(t_{n+1}-s\right) \Omega\right) B d W_{s}-\Omega^{-1} \sin (h \Omega) B \Delta W_{n} \in \mathbb{R}^{d} \\
& d_{n}^{2}=\int_{t_{n}}^{t_{n+1}} \cos \left(\left(t_{n+1}-s\right) \Omega\right) B d W_{s}-\cos (h \Omega) B \Delta W_{n} \in \mathbb{R}^{d}
\end{aligned}
$$

By Lemma 1 and properties of the Ito integral, we have the following estimates for the defects

$$
\mathbb{E}\left[d_{n}^{1}\right]=\mathbb{E}\left[d_{n}^{2}\right]=0, \mathbb{E}\left[\left\|d_{n}^{1}\right\|^{2}\right]=\mathcal{O}\left(\varepsilon^{2} h\right), \mathbb{E}\left[\left\|d_{n}^{2}\right\|^{2}\right]=\mathcal{O}(h) .
$$


We now define the errors $e_{n}^{j}=X_{t_{n}}^{j}-X_{n}^{j} \in \mathbb{R}^{d}$, for $j=1,2$. A subtraction of (4) from the definition of the scheme gives us

$$
E_{n+1}=R \cdot E_{n}+d_{n}
$$

where $E_{n}=\left(\begin{array}{c}e_{n}^{1} \\ e_{n}^{2}\end{array}\right)$ and $d_{n}=\left(\begin{array}{l}d_{n}^{1} \\ d_{n}^{2}\end{array}\right)$. A recursion leads to the following formula for the errors:

$$
E_{n+1}=R^{n+1} E_{0}+\sum_{j=0}^{n} R^{n-j} d_{j}=\sum_{j=0}^{n} R^{n-j} d_{j}
$$

with the matrix $R^{n-j}=\left(\begin{array}{cc}\cos ((n-j) h \Omega) & \Omega^{-1} \sin ((n-j) h \Omega) \\ -\Omega \sin ((n-j) h \Omega) & \cos ((n-j) h \Omega)\end{array}\right)$.

All together and the independence of the Wiener increments give:

$$
\begin{aligned}
\mathbb{E}\left[\left\|e_{n+1}^{1}\right\|^{2}\right]= & \mathbb{E}\left[\left\|\sum_{j=0}^{n}\left(\cos ((n-j) h \Omega) d_{j}^{1}+\Omega^{-1} \sin ((n-j) h \Omega) d_{j}^{2}\right)\right\|^{2}\right] \\
= & \sum_{j=0}^{n} \mathbb{E}\left[\left\|\cos ((n-j) h \Omega) d_{j}^{1}+\Omega^{-1} \sin ((n-j) h \Omega) d_{j}^{2}\right\|^{2}\right] \\
& +2 \sum_{j<\ell} \mathbb{E}\left[\left(\cos ((n-j) h \Omega) d_{j}^{1}+\Omega^{-1} \sin ((n-j) h \Omega) d_{j}^{2}\right)^{T}\right. \\
& \left.\left(\cos ((n-\ell) h \Omega) d_{\ell}^{1}+\Omega^{-1} \sin ((n-\ell) h \Omega) d_{\ell}^{2}\right)\right] \\
= & \sum_{j=0}^{n} \mathbb{E}\left[\left\|\cos ((n-j) h \Omega) d_{j}^{1}+\Omega^{-1} \sin ((n-j) h \Omega) d_{j}^{2}\right\|^{2}\right] .
\end{aligned}
$$

Once again using the Ito isometry, we obtain

$$
\begin{aligned}
& \mathbb{E}\left[\left\|\cos ((n-j) h \Omega) d_{j}^{1}+\Omega^{-1} \sin ((n-j) h \Omega) d_{j}^{2}\right\|^{2}\right] \\
& =\mathbb{E}\left[\int_{t_{j}}^{t_{j+1}} \| \cos ((n-j) h \Omega) \Omega^{-1}\left(\sin \left(\left(t_{j+1}-s\right) \Omega\right)-\sin (h \Omega)\right) B\right. \\
& \left.\quad+\Omega^{-1} \sin ((n-j) h \Omega)\left(\cos \left(\left(t_{j+1}-s\right) \Omega\right)-\cos (h \Omega)\right) B \|^{2} d s\right] .
\end{aligned}
$$

Similar to the proof of Lemma 1, we can bound the above term with:

$$
\mathbb{E}\left[\left\|\cos ((n-j) h \Omega) d_{j}^{1}+\Omega^{-1} \sin ((n-j) h \Omega) d_{j}^{2}\right\|^{2}\right]=\mathcal{O}\left(h \varepsilon^{2}\right)
$$


Finally summing up, using the fact that $h / \varepsilon \geq c_{0}$ and that $n h \leq T$ give the desired bound for the position component:

$$
\mathbb{E}\left[\left\|e_{n}^{1}\right\|^{2}\right]=\mathbb{E}\left[\left\|X_{n}^{1}-X_{n h}^{1}\right\|^{2}\right] \leq C T \varepsilon^{2} \leq C T h^{2} .
$$

The bound for the velocity component is obtained in a similar way.

It is now time to consider the nonlinear case (2). Our convergence proof heavily relies on the main result given in [4] for the corresponding deterministic case. For ease of reading we first recall the main theorem of [4]:

Theorem 1 (Theorem 1 in [4]) Let us consider the deterministic problem $\ddot{x}+A x=$ $g(x)$, with A a positive semi-definite symmetric matrix, and the numerical solution given by (3) with $B=0$. Under the following assumptions:

1. Suppose that $g, g^{\prime}$ and $g^{\prime \prime}$ are bounded.

2. Assume that the exact solution satisfies $\frac{1}{2}\|\dot{x}(t)\|^{2}+\frac{1}{2}\|\Omega x(t)\|^{2} \leq \frac{1}{2} K^{2}$ for $0 \leq$ $t \leq T$.

3. The filter functions have to satisfy the following assumptions:

$$
\max _{\xi \geq 0}|\chi(\xi)| \leq M_{1}, \text { for } \chi=\phi, \psi, \psi_{0}, \psi_{1}
$$

for some constant $M_{1}$. There exist further constants $M_{2}, M_{3}, M_{4}, M_{5}, M_{6}$ and $M_{7}$ such that

$$
\begin{aligned}
& \max _{\xi \geq 0}\left|\frac{\phi(\xi)-1}{\xi}\right| \leq M_{2}, \\
& \max _{\xi \geq 0}\left|\frac{1}{\sin \left(\frac{\xi}{2}\right)}\left(\operatorname{sinc}^{2}\left(\frac{\xi}{2}\right)-\psi(\xi)\right)\right| \leq M_{3}, \\
& \max _{\xi \geq 0}\left|\frac{1}{\xi \sin \left(\frac{\xi}{2}\right)}(\operatorname{sinc}(\xi)-\chi(\xi))\right| \leq M_{4}, \quad \chi=\phi, \psi_{0}, \psi_{1}, \\
& \max _{\xi \geq 0}|\xi \psi(\xi)| \leq M_{5}, \quad \max _{\xi \geq 0}\left|\frac{\xi}{\sin \left(\frac{\xi}{2}\right)}\left(\operatorname{sinc}^{2}\left(\frac{\xi}{2}\right)-\psi(\xi)\right)\right| \leq M_{6} \quad \text { and } \\
& \max _{\xi \geq 0}\left|\frac{1}{\sin \left(\frac{\xi}{2}\right)}\left(\operatorname{sinc}(\xi)-\psi_{i}(\xi)\right)\right| \leq M_{7}, \quad i=0,1 .
\end{aligned}
$$

Moreover, let us define $M:=\max _{i=1, \ldots, 7} M_{i}$.

If Eq. (5)-(8) are verified then the error in the position satisfies

$$
\left\|x_{t_{n}}-x_{n}\right\| \leq C h^{2}, \quad 0 \leq t_{n}=n h \leq T .
$$

The constant $C$ only depends on $T, K, M_{1}, \ldots, M_{4},\|g\|,\left\|g^{\prime}\right\|$ and $\left\|g^{\prime \prime}\right\|$, but not on $\varepsilon$. If, in addition, (9) and (10) are satisfied, then 


$$
\left\|\dot{x}_{t_{n}}-\dot{x}_{n}\right\| \leq \tilde{C} h, \quad 0 \leq t_{n}=n h \leq T .
$$

The constant $\tilde{C}$ only depends on $T, K, M_{1}, \ldots, M_{4},\|g\|,\left\|g^{\prime}\right\|$ and $\left\|g^{\prime \prime}\right\|$, but not on $\varepsilon$.

As already noted in [4], these error bounds are independent of the dimension of the problem and the constant $C$ (resp. $\tilde{C}$ ) does not depend on the large norm of the matrix present in our problem. These properties are very desirable, for example if the system (1) results from a semi-discretisation of a nonlinear wave equation. In this case, the bounds are independent of the mesh size used for the spatial discretisation.

Example 3 The following choices for the filter functions fulfill all the above conditions, [4]:

$\phi(\xi)=\operatorname{sinc}(\xi), \psi(\xi)=\operatorname{sinc}^{3}(\xi), \psi_{0}(\xi)=\cos (\xi) \operatorname{sinc}^{2}(\xi), \quad$ and $\quad \psi_{1}(\xi)=\operatorname{sinc}^{2}(\xi)$

We can now give the main result of this section:

Theorem 2 Under the assumptions of Proposition 1 and Theorem 1, the global meansquare errors satisfy

$$
\begin{aligned}
& \left(\mathbb{E}\left[\left\|X_{n}^{1}-X_{n h}^{1}\right\|^{2}\right]\right)^{1 / 2} \leq C\left(\varepsilon^{2}+h^{4}\right)^{1 / 2} \leq C h \\
& \left(\mathbb{E}\left[\left\|X_{n}^{2}-X_{n h}^{2}\right\|^{2}\right]\right)^{1 / 2} \leq C T^{1 / 2} \quad \text { for } n h \leq T,
\end{aligned}
$$

where the constant $C$ only depends on $T, K, M_{1}, \ldots, M_{4},\|g\|,\left\|g^{\prime}\right\|$ and $\left\|g^{\prime \prime}\right\|$, but is independent of $\varepsilon, h$ and $n$ with $n h \leq T$.

Proof Let us introduce the following notations for the position component:

$$
\begin{aligned}
& q_{1}:= \Omega^{-1} \sum_{j=0}^{n-1}(\cos (j h \Omega) \sin (h \Omega)+\sin (j h \Omega) \cos (h \Omega)) B \triangle W_{n-j-1}, \\
& \hat{q}_{1}:=\Omega^{-1} \sum_{j=0}^{n-1}\left(\cos (j h \Omega) \int_{t_{n-j-1}}^{t_{n-j}} \sin \left(\left(t_{n-j}-s\right) \Omega\right) B d W_{s}\right. \\
&\left.+\sin (j h \Omega) \int_{t_{n-j-1}}^{t_{n-j}} \cos \left(\left(t_{n-j}-s\right) \Omega\right) B d W_{s}\right)
\end{aligned}
$$




$$
\begin{aligned}
r_{1}:= & \sum_{j=0}^{n-1}\left(\cos (j h \Omega) \frac{h}{2} h \Psi g_{n-j-1}+\Omega^{-1} \sin (j h \Omega) \frac{h}{2} \Psi_{1} g_{n-j}\right. \\
& \left.+\Omega^{-1} \sin (j h \Omega) \frac{h}{2} \Psi_{0} g_{n-j-1}\right), \\
\hat{r}_{1}:= & \Omega^{-1} \sum_{j=0}^{n-1}\left(\cos (j h \Omega) \int_{t_{n-j-1}}^{t_{n-j}} \sin \left(\left(t_{n-j}-s\right) \Omega\right) g\left(X_{s}^{1}\right) d s\right. \\
& +\sin (j h \Omega) \int_{t_{n-j-1}}^{t_{n-j}} \cos \left(\left(t_{n-j}-s\right) \Omega\right) g\left(X_{s}^{1}\right) d s
\end{aligned}
$$

where $g_{n}=g\left(\Phi X_{n}^{1}\right)$. For the velocity component, we set

$$
\begin{aligned}
& q_{2}:=\sum_{j=0}^{n-1}(\sin (j h \Omega) \sin (h \Omega)-\cos (j h \Omega) \cos (h \Omega)) B \triangle W_{n-j-1}, \\
& \hat{q}_{2}:=-\sum_{j=0}^{n-1}\left(\sin (j h \Omega) \int_{t_{n-j-1}}^{t_{n-j}} \sin \left(\left(t_{n-j}-s\right) \Omega\right) B d W_{s}\right. \\
& \left.+\cos (j h \Omega) \int_{t_{n-j-1}}^{t_{n-j}} \cos \left(\left(t_{n-j}-s\right) \Omega\right) B d W_{s}\right), \\
& r_{2}:=-\sum_{j=0}^{n-1}\left(\sin (j h \Omega) \frac{h}{2} h \Omega \Psi g_{n-j-1}-\cos (j h \Omega) \frac{h}{2} \Psi_{1} g_{n-j}\right. \\
& \left.-\cos (j h \Omega) \frac{h}{2} \Psi_{0} g_{n-j-1}\right), \\
& \hat{r}_{2}:=-\sum_{j=0}^{n-1}\left(\sin (j h \Omega) \int_{t_{n-j-1}}^{t_{n-j}} \sin \left(\left(t_{n-j}-s\right) \Omega\right) g\left(X_{s}^{1}\right) d s\right. \\
& \left.-\cos (j h \Omega) \int_{t_{n-j-1}}^{t_{n-j}} \cos \left(\left(t_{n-j}-s\right) \Omega\right) g\left(X_{s}^{1}\right) d s\right) \text {. }
\end{aligned}
$$

The $n$th iterate of the numerical scheme (3) thus reads

$$
\begin{aligned}
& X_{n}^{1}=\cos (n h \Omega) X_{0}^{1}+\Omega^{-1} \sin (n h \Omega) X_{0}^{2}+q_{1}+r_{1} \\
& X_{n}^{2}=-\Omega \sin (n h \Omega) X_{0}^{1}+\cos (n h \Omega) X_{0}^{2}+q_{2}+r_{2},
\end{aligned}
$$

and for the exact solution we have 


$$
\begin{aligned}
& X_{n h}^{1}=\cos (n h \Omega) X_{0}^{1}+\Omega^{-1} \sin (n h \Omega) X_{0}^{2}+\hat{q}_{1}+\hat{r}_{1} \\
& X_{n h}^{2}=-\Omega \sin (n h \Omega) X_{0}^{1}+\cos (n h \Omega) X_{0}^{2}+\hat{q}_{2}+\hat{r}_{2} .
\end{aligned}
$$

We thus get, for the global mean-square error in the position:

$$
\mathbb{E}\left[\left\|X_{n}^{1}-X_{n h}^{1}\right\|^{2}\right]=\mathbb{E}\left[\left\|q_{1}+r_{1}-\hat{q}_{1}-\hat{r}_{1}\right\|^{2}\right] \leq 2 \mathbb{E}\left[\left\|q_{1}-\hat{q}_{1}\right\|^{2}\right]+2 \mathbb{E}\left[\left\|r_{1}-\hat{r}_{1}\right\|^{2}\right] .
$$

The first term on the right-hand side is the global mean-square error in the linear case (see Proposition 1) and the second one is the global error in the deterministic case (see Theorem 1). We finally obtain

$$
\mathbb{E}\left[\left\|X_{n}^{1}-X_{n h}^{1}\right\|^{2}\right] \leq 2 \mathbb{E}\left[\left\|q_{1}-\hat{q}_{1}\right\|^{2}\right]+2 \mathbb{E}\left[\left\|r_{1}-\hat{r}_{1}\right\|^{2}\right] \leq C\left(\varepsilon^{2}+h^{4}\right) .
$$

The bound for the velocity component is obtained in a similar way.

We conclude this section by mentioning the fact, that for fixed $\varepsilon$, the numerical schemes still converge as the step size goes to zero.

Remark 3 In this case Taylor expansions of the expressions in the proof of Lemma 1 can be used to determine the mean-square errors after one step. We have

$$
\begin{aligned}
\mathbb{E}\left[\left\|X_{1}^{1}-X_{h}^{1}\right\|^{2}\right] & \leq\|B\|^{2}\left\|\Omega^{-1}\right\|^{2} \int_{0}^{h}\|\sin (h \Omega)-\sin ((h-s) \Omega)\|^{2} d s \\
& =\|B\|^{2}\left\|\Omega^{-1}\right\|^{2} \int_{0}^{h}\left\|\sin (h \Omega)-\sum_{n \geq 0} \sin ^{(n)}(h \Omega) \frac{(-s \Omega)^{n}}{n !}\right\|^{2} d s \\
& \leq C h^{3}
\end{aligned}
$$

and similarly

$$
\mathbb{E}\left[\left\|X_{1}^{2}-X_{h}^{2}\right\|^{2}\right] \leq C h^{5}
$$

Hence, for a fixed $\varepsilon$, we obtain, instead of Theorem 2, the following error bounds

$$
\begin{aligned}
& \left(\mathbb{E}\left[\left\|X_{n}^{1}-X_{n h}^{1}\right\|^{2}\right]\right)^{1 / 2} \leq C h \\
& \left(\mathbb{E}\left[\left\|X_{n}^{2}-X_{n h}^{2}\right\|^{2}\right]\right)^{1 / 2} \leq C h \quad \text { for } n h \leq T .
\end{aligned}
$$

\section{Convergence in the second moment}

In this section, we will derive error bounds for the stochastic trigonometric methods in the second moment. Let us quickly restate the definition. 
Definition 2 A numerical method $\left\{Y_{n}\right\}$ with step size $h$ converges in the second moment with order $\gamma>0$ to the solution $Y_{t_{n}}$ of an SDE at time $t_{n}=n h$ if $\gamma$ is the largest value such that there exists a positive constant $C$, which does not depend on $h$, and an $h_{0}>0$ such that

$$
\left|\mathbb{E}\left[\left\|Y_{t_{n}}\right\|^{2}\right]-\mathbb{E}\left[\left\|Y_{n}\right\|^{2}\right]\right| \leq C h^{\gamma}
$$

for all $h \in\left(0, h_{0}\right)$.

We first show that the local error in the second moment for the position of our stochastic trigonometric integrator is of order $\mathcal{O}\left(h^{3}\right)$ for linear problems.

Lemma 2 Consider the numerical solution of (1) with $g \equiv 0$ by method (3) with a step size $h \leq h_{0}$ (with a sufficiently small $h_{0}$ independent of $\varepsilon$ ) for which $h / \varepsilon \geq c_{0}>0$ holds. The errors in the second moment after one step of the numerical scheme satisfy

$$
\begin{aligned}
& \left|\mathbb{E}\left[\left\|X_{1}^{1}\right\|^{2}\right]-\mathbb{E}\left[\left\|X_{h}^{1}\right\|^{2}\right]\right| \leq C h \varepsilon^{2} \leq C h^{3} \\
& \left|\mathbb{E}\left[\left\|X_{1}^{2}\right\|^{2}\right]-\mathbb{E}\left[\left\|X_{h}^{2}\right\|^{2}\right]\right| \leq C \varepsilon \leq C h,
\end{aligned}
$$

where the constant $C$ is independent of $\varepsilon$ and $h$. That is, the local errors are of order (at least) one uniformly in the frequencies.

Proof Let us start with the error in the position. By definitions of the scheme, of the exact solution, and using some properties of the Wiener increments and the Ito isometry, we obtain

$$
\begin{aligned}
\left|\mathbb{E}\left[\left\|X_{1}^{1}\right\|^{2}\right]-\mathbb{E}\left[\left\|X_{h}^{1}\right\|^{2}\right]\right|= & \mid \mathbb{E}\left[\left\|\Omega^{-1} \sin (h \Omega) B \Delta W_{0}\right\|^{2}\right] \\
& -\mathbb{E}\left[\left\|\int_{0}^{h} \Omega^{-1} \sin ((h-s) \Omega) B d W_{s}\right\|^{2}\right] \mid \\
= & \mid \mathbb{E}\left[\left\|\Omega^{-1} \sin (h \Omega) B \Delta W_{0}\right\|^{2}\right] \\
& -\int_{0}^{h}\left\|\Omega^{-1} \sin ((h-s) \Omega) B\right\|^{2} d s \mid \leq C h \varepsilon^{2} .
\end{aligned}
$$

Similarly, concerning the error in the second component, we have

$$
\begin{aligned}
\left|\mathbb{E}\left[\left\|X_{1}^{2}\right\|^{2}\right]-\mathbb{E}\left[\left\|X_{h}^{2}\right\|^{2}\right]\right|= & \mid \mathbb{E}\left[\left\|\cos (h \Omega) B \Delta W_{0}\right\|^{2}\right] \\
& -\mathbb{E}\left[\left\|\int_{0}^{h} \cos ((h-s) \Omega) B d W_{s}\right\|^{2}\right] \mid
\end{aligned}
$$




$$
\begin{aligned}
= & \mid \mathbb{E}\left[\left\|\cos (h \Omega) B \Delta W_{0}\right\|^{2}\right] \\
& -\int_{0}^{h}\|\cos ((h-s) \Omega) B\|^{2} d s \mid \leq C h .
\end{aligned}
$$

We can now derive global error bounds for the linear case. We obtain the following result.

Proposition 2 Consider the numerical solution of (1) with $g \equiv 0$ by method (3) with a step size $h \leq h_{0}$ (with a sufficiently small $h_{0}$ independent of $\varepsilon$ ) for which $h / \varepsilon \geq c_{0}>0$ holds. The errors in the second moment of the numerical scheme satisfy

$$
\begin{aligned}
& \left|\mathbb{E}\left[\left\|X_{n}^{1}\right\|^{2}\right]-\mathbb{E}\left[\left\|X_{n h}^{1}\right\|^{2}\right]\right| \leq C \varepsilon^{2} T \leq C h^{2} \\
& \left|\mathbb{E}\left[\left\|X_{n}^{2}\right\|^{2}\right]-\mathbb{E}\left[\left\|X_{n h}^{2}\right\|^{2}\right]\right| \leq C T \quad \text { for } n h \leq T,
\end{aligned}
$$

where the constant $C$ is independent of $\varepsilon, h$ and $n$ with $n h \leq T$. That is, the global error in the position component is of order two uniformly in the frequencies.

Proof In order to determine the global order of convergence in the position, we study the expression $\left|\mathbb{E}\left[\left\|X_{n}^{1}\right\|^{2}\right]-\mathbb{E}\left[\left\|X_{n h}^{1}\right\|^{2}\right]\right|$. Using the notations of Theorem 2, we obtain

$$
\begin{aligned}
\left|\mathbb{E}\left[\left\|X_{n}^{1}\right\|^{2}\right]-\mathbb{E}\left[\left\|X_{n h}^{1}\right\|^{2}\right]\right|= & \mid \mathbb{E}\left[\left\|\cos (n h \Omega) X_{0}^{1}+\Omega^{-1} \sin (n h \Omega) X_{0}^{2}+q_{1}\right\|^{2}\right] \\
& -\mathbb{E}\left[\left\|\cos (n h \Omega) X_{0}^{1}+\Omega^{-1} \sin (n h \Omega) X_{0}^{2}+\hat{q}_{1}\right\|^{2}\right] \mid .
\end{aligned}
$$

Using properties of the Wiener increments and of the Ito integral, we get

$$
\begin{aligned}
\left|\mathbb{E}\left[\left\|X_{n}^{1}\right\|^{2}\right]-\mathbb{E}\left[\left\|X_{n h}^{1}\right\|^{2}\right]\right|=\left|\mathbb{E}\left[\left\|q_{1}\right\|^{2}\right]-\mathbb{E}\left[\left\|\hat{q}_{1}\right\|^{2}\right]\right| \\
=\mid \sum_{j=0}^{n-1} \mathbb{E}\left[\left\|\Omega^{-1}(\cos (j h \Omega) \sin (h \Omega)+\sin (j h \Omega) \cos (h \Omega)) B \Delta W_{n-j-1}\right\|^{2}\right] \\
\quad-\sum_{j=0_{t_{n-j-1}}}^{n-1} \| \Omega^{-1}\left(\cos (j h \Omega) \sin \left(\left(t_{n-j}-s\right) \Omega\right)\right. \\
\left.\quad+\sin (j h \Omega) \cos \left(\left(t_{n-j}-s\right) \Omega\right)\right) B \|^{2} d s \mid \\
\leq C \varepsilon^{2} n h \leq C \varepsilon^{2} T .
\end{aligned}
$$


Analogously we get for the global order of convergence in the velocity

$$
\begin{aligned}
\mid \mathbb{E} & {\left[\left\|X_{n}^{2}\right\|^{2}\right]-\mathbb{E}\left[\left\|X_{n h}^{2}\right\|^{2}\right]|=| \mathbb{E}\left[\left\|q_{2}\right\|^{2}\right]-\mathbb{E}\left[\left\|\hat{q}_{2}\right\|^{2}\right] \mid } \\
= & \mid \sum_{j=0}^{n-1} \mathbb{E}\left[\left\|(\sin (j h \Omega) \sin (h \Omega)-\cos (j h \Omega) \cos (h \Omega)) B \Delta W_{n-j-1}\right\|^{2}\right] \\
& +\sum_{j=0}^{n-1} \int_{t_{n-j-1}}^{t_{n-j}}\left\|\left(\sin (j h \Omega) \sin \left(\left(t_{n-j}-s\right) \Omega\right)+\cos (j h \Omega) \cos \left(\left(t_{n-j}-s\right) \Omega\right)\right) B\right\|^{2} d s \mid \\
\leq & C n h \leq C T
\end{aligned}
$$

or the boundedness of the global error in the velocity.

We can now state the main result of this section:

Theorem 3 Under the assumptions of Proposition 2 and Theorem 1, the global errors in the second moment satisfy

$$
\begin{aligned}
& \left|\mathbb{E}\left[\left\|X_{n}^{1}\right\|^{2}\right]-\mathbb{E}\left[\left\|X_{n h}^{1}\right\|^{2}\right]\right| \leq C\left(\varepsilon^{2}+(h+\varepsilon)^{2}+h^{2}+\left(h \varepsilon+\varepsilon^{2}\right)\right) \leq C h^{2} \\
& \left|\mathbb{E}\left[\left\|X_{n}^{2}\right\|^{2}\right]-\mathbb{E}\left[\left\|X_{n h}^{2}\right\|^{2}\right]\right| \leq C(T+h) \leq C T \quad \text { for } n h \leq T,
\end{aligned}
$$

where the constant $C$ only depends on $T, K, M_{1}, \ldots, M_{4},\|g\|,\left\|g^{\prime}\right\|$ and $\left\|g^{\prime \prime}\right\|$, but is independent of $\varepsilon, h$ and $n$ with $n h \leq T$.

Proof Let us first define the following quantities

$p_{1}=\cos (n h \Omega) X_{0}^{1}+\Omega^{-1} \sin (n h \Omega) X_{0}^{2} \quad$ and $\quad p_{2}=-\Omega \sin (n h \Omega) X_{0}^{1}+\cos (n h \Omega) X_{0}^{2}$

so that the numerical scheme can be written as

$$
X_{n}^{1}=p_{1}+q_{1}+r_{1} \text { and } X_{n}^{2}=p_{2}+q_{2}+r_{2}
$$

For the error in the second moment for the position, we thus obtain

$$
\begin{aligned}
\left|\mathbb{E}\left[\left\|X_{n}^{1}\right\|^{2}\right]-\mathbb{E}\left[\left\|X_{n h}^{1}\right\|^{2}\right]\right|= & \mid \mathbb{E}\left[2 p_{1}^{T} q_{1}+2 p_{1}^{T} r_{1}+q_{1}^{T} q_{1}+2 q_{1}^{T} r_{1}+r_{1}^{T} r_{1}\right. \\
& \left.-\hat{q}_{1}^{T} \hat{q}_{1}-\hat{r}_{1}^{T} \hat{r}_{1}-2 p_{1}^{T} \hat{r}_{1}-2 p_{1}^{T} \hat{q}_{1}-2 \hat{q}_{1}^{T} \hat{r}_{1}\right] \mid \\
\leq & \left|\mathbb{E}\left[\left\|q_{1}\right\|^{2}-\left\|\hat{q}_{1}\right\|^{2}\right]\right|+\left|\mathbb{E}\left[\left\|r_{1}\right\|^{2}-\left\|\hat{r}_{1}\right\|^{2}\right]\right| \\
& +2 \mathbb{E}\left[\left|p_{1}^{T}\left(r_{1}-\hat{r}_{1}\right)\right|\right]+2\left|\mathbb{E}\left[q_{1}^{T} r_{1}-\hat{q}_{1}^{T} \hat{r}_{1}\right]\right|
\end{aligned}
$$

due to the fact that $\mathbb{E}\left[\Delta W_{n-j-1}\right]=0$ and due to the martingale property of the Ito integral. We will now estimate the four terms on the right. Thanks to Proposition 2, we have

$$
\left|\mathbb{E}\left[\left\|q_{1}\right\|^{2}-\left\|\hat{q}_{1}\right\|^{2}\right]\right| \leq C T \varepsilon^{2} .
$$


Using the bounds for the function $g$, the bounds for the filter functions and the triangle inequality, we get

$$
\begin{aligned}
\left|\mathbb{E}\left[\left\|r_{1}\right\|^{2}-\left\|\hat{r}_{1}\right\|^{2}\right]\right| & \leq \mathbb{E}\left[\left\|r_{1}\right\|^{2}\right]+\mathbb{E}\left[\left\|\hat{r}_{1}\right\|^{2}\right] \leq\left(C n\left(h^{2}+h \varepsilon\right)\right)^{2}+(C n h \varepsilon)^{2} \\
& \leq C T^{2}(h+\varepsilon)^{2} .
\end{aligned}
$$

The Cauchy-Schwarz inequality and Theorem 1 give us the bounds

$$
2 \mathbb{E}\left[\left|p_{1}^{T}\left(r_{1}-\hat{r}_{1}\right)\right|\right] \leq 2\left\|p_{1}\right\| \mathbb{E}\left[\left\|r_{1}-\hat{r}_{1}\right\|\right] \leq C h^{2}
$$

For the last term, we obtain

$$
\begin{aligned}
\mathbb{E}\left[\left|q_{1}^{T} r_{1}\right|\right. & \leq \mathbb{E}\left[\left\|r_{1}\right\|\left\|q_{1}\right\|\right] \leq C T(h+\varepsilon) \mathbb{E}\left[\left\|q_{1}\right\|\right] \leq C T(h+\varepsilon) \sqrt{\mathbb{E}\left[\left\|q_{1}\right\|^{2}\right]} \\
& \leq C T(h+\varepsilon) \varepsilon
\end{aligned}
$$

thanks to the Cauchy-Schwarz inequality, the bounds for the function $g$ and Proposition 2. This finally gives us

$$
2\left|\mathbb{E}\left[q_{1}^{T} r_{1}-\hat{q}_{1}^{T} \hat{r}_{1}\right]\right| \leq C T\left(h \varepsilon+\varepsilon^{2}\right)
$$

All together, we obtain the bounds for the position

$$
\left|\mathbb{E}\left[\left\|X_{n}^{1}\right\|^{2}\right]-\mathbb{E}\left[\left\|X_{n h}^{1}\right\|^{2}\right]\right| \leq C\left(\varepsilon^{2}+(h+\varepsilon)^{2}+h^{2}+\left(h \varepsilon+\varepsilon^{2}\right)\right) .
$$

For the velocity component we consider the expression

$$
\begin{aligned}
\left|\mathbb{E}\left[\left\|X_{n}^{2}\right\|^{2}\right]-\mathbb{E}\left[\left\|X_{n h}^{2}\right\|^{2}\right]\right| \leq & \left|\mathbb{E}\left[\left\|q_{2}\right\|^{2}-\left\|\hat{q}_{2}\right\|^{2}\right]\right|+\left|\mathbb{E}\left[\left\|r_{2}\right\|^{2}-\left\|\hat{r}_{2}\right\|^{2}\right]\right| \\
& +2 \mathbb{E}\left[\left|p_{2}^{T}\left(r_{2}-\hat{r}_{2}\right)\right|\right]+2\left|\mathbb{E}\left[q_{2}^{T} r_{2}-\hat{q}_{2}^{T} \hat{r}_{2}\right]\right| .
\end{aligned}
$$

Similarly, we get the following bounds for the individual terms on the right

$$
\begin{aligned}
\left|\mathbb{E}\left[\left\|q_{2}\right\|^{2}-\left\|\hat{q}_{2}\right\|^{2}\right]\right| & =\mathcal{O}(T), & & \left|\mathbb{E}\left[\left\|r_{2}\right\|^{2}-\left\|\hat{r}_{2}\right\|^{2}\right]\right|=\mathcal{O}(T), \\
2 \mathbb{E}\left[\left|p_{2}^{T}\left(r_{2}-\hat{r}_{2}\right)\right|\right] & =\mathcal{O}(h), & & 2\left|\mathbb{E}\left[q_{2}^{T} r_{2}-\hat{q}_{2}^{T} \hat{r}_{2}\right]\right|=\mathcal{O}(T) .
\end{aligned}
$$

This yields for the whole expression

$$
\left|\mathbb{E}\left[\left\|X_{n}^{2}\right\|^{2}\right]-\mathbb{E}\left[\left\|X_{n h}^{2}\right\|^{2}\right]\right| \leq C(T+h)
$$

or the boundedness of the global error in the second moment for the velocity.

As in the mean-square case, we conclude this section by looking at the convergence for a fixed $\varepsilon$. 
Remark 4 We thus fix $\varepsilon$ and let $h$ tend to zero in order to illustrate the convergence behaviour. Therefore Taylor expansions of the expressions in the proof of Lemma 2 can be used to determine the errors in the second moment after one step.

$$
\begin{aligned}
\left|\mathbb{E}\left[\left\|X_{1}^{1}\right\|^{2}\right]-\mathbb{E}\left[\left\|X_{h}^{1}\right\|^{2}\right]\right|= & \mid \mathbb{E}\left[\left\|\Omega^{-1} \sin (h \Omega) B \Delta W_{0}\right\|^{2}\right] \\
& -\int_{0}^{h}\left\|\Omega^{-1} \sin ((h-s) \Omega) B\right\|^{2} d s \mid \\
= & \mid \mathbb{E}\left[\left\|\Omega^{-1} \sin (h \Omega) B \Delta W_{0}\right\|^{2}\right] \\
& -\int_{0}^{h}\left\|\Omega^{-1} \sum_{n \geq 0} \sin ^{(n)}(h \Omega) \frac{(-s \Omega)^{n}}{n !} B\right\|^{2} d s \mid \\
\leq & C h^{3}
\end{aligned}
$$

and similarly

$$
\left|\mathbb{E}\left[\left\|X_{1}^{2}\right\|^{2}\right]-\mathbb{E}\left[\left\|X_{h}^{2}\right\|^{2}\right]\right| \leq C h .
$$

Hence, for a fixed $\varepsilon$, we obtain, instead of Theorem 3, the following error bounds

$$
\begin{aligned}
& \left|\mathbb{E}\left[\left\|X_{n}^{1}\right\|^{2}\right]-\mathbb{E}\left[\left\|X_{n h}^{1}\right\|^{2}\right]\right| \leq C h \\
& \left|\mathbb{E}\left[\left\|X_{n}^{2}\right\|^{2}\right]-\mathbb{E}\left[\left\|X_{n h}^{2}\right\|^{2}\right]\right| \leq C T \quad \text { for } n h \leq T .
\end{aligned}
$$

\section{Growth rate of the expected energy}

The exact solution of our problem (2) with a smooth gradient nonlinearity $g(x)=$ $-\nabla U(x)$ has the following interesting geometric property:

Applying Ito's formula, it is known (see for example [15]) that the expected value of the energy has a linear growth in time:

$$
\begin{aligned}
\mathbb{E}[ & \left.\frac{1}{2}\left(\left\|X_{t}^{2}\right\|^{2}+\left\|\Omega X_{t}^{1}\right\|^{2}\right)+U\left(X_{t}^{1}\right)\right] \\
& =\frac{1}{2}\left(\left\|y_{0}\right\|^{2}+\left\|\Omega x_{0}\right\|^{2}\right)+U\left(x_{0}\right)+\frac{\operatorname{Tr}\left(B B^{T}\right)}{2} t,
\end{aligned}
$$

where $X_{0}^{1}=x_{0}$ and $X_{0}^{2}=y_{0}$ are the initial position, resp. velocity for the problem (2).

In a geometric numerical integration approach (see the monographs [5,12] for the deterministic case), one would seek numerical schemes that reproduce geometric properties of the exact solution of the differential equation. We will see that the proposed schemes capture almost the correct energy growth rate. 
In order to show the almost-linear growth rate of the expected value of the energy for the numerical solution, we need the following lemma:

Lemma 3 Under the assumptions of Theorem 2 we have

$$
\mathbb{E}\left[\left\|\Omega\left(r_{1}-\hat{r}_{1}\right)\right\|\right] \leq C h \text { for } n h \leq T,
$$

where $r_{1}$ and $\hat{r}_{1}$ are defined in the proof of Theorem 2.

Proof Writing down the definitions of $r_{1}$ and $\hat{r}_{1}$ we get

$$
\begin{aligned}
& \left\|\Omega\left(r_{1}-\hat{r}_{1}\right)\right\|=\| \sum_{j=0}^{n-1}\left(\cos (j h \Omega) \frac{h}{2} h \Omega \Psi g_{n-j-1}\right. \\
& \left.+\sin (j h \Omega) \frac{h}{2} \Psi_{1} g_{n-j}+\sin (j h \Omega) \frac{h}{2} \Psi_{0} g_{n-j-1}\right) \\
& -\sum_{j=0}^{n-1}\left(\cos (j h \Omega) \int_{t_{n-j-1}}^{t_{n-j}} \sin \left(\left(t_{n-j}-s\right) \Omega\right) g\left(X_{s}^{1}\right) d s\right. \\
& \left.+\sin (j h \Omega) \int_{t_{n-j-1}}^{t_{n-j}} \cos \left(\left(t_{n-j}-s\right) \Omega\right) g\left(X_{s}^{1}\right) d s\right) \| \\
& =\left\|\sum_{j=0}^{n-1} \Omega \cos (j h \Omega) e_{n-j-1}+\sum_{j=0}^{n-1} \sin (j h \Omega) e_{n-j-1}^{\prime}\right\| \\
& \leq\left\|\sum_{j=0}^{n-1} \Omega \cos (j h \Omega) e_{n-j-1}\right\|+\left\|\sum_{j=0}^{n-1} \sin (j h \Omega) e_{n-j-1}^{\prime}\right\|,
\end{aligned}
$$

where

$$
\begin{aligned}
& e_{n-j-1}=\frac{h}{2} h \Psi g_{n-j-1}-\int_{t_{n-j-1}}^{t_{n-j}} \Omega^{-1} \sin \left(\left(t_{n-j}-s\right) \Omega\right) g\left(X_{s}^{1}\right) d s \text { and } \\
& e_{n-j-1}^{\prime}=\frac{h}{2} \Psi_{1} g_{n-j}+\frac{h}{2} \Psi_{0} g_{n-j-1}-\int_{t_{n-j-1}}^{t_{n-j}} \cos \left(\left(t_{n-j}-s\right) \Omega\right) g\left(X_{s}^{1}\right) d s .
\end{aligned}
$$


We will now use some lemmas from [4] to estimate the above expressions. We begin by the term with $e_{n-j-1}$. We write

$$
\begin{aligned}
e_{n-j-1}= & \frac{h}{2} h \Psi g\left(\Phi X_{t_{n-j-1}}^{1}\right)-\int_{t_{n-j-1}}^{t_{n-j}} \Omega^{-1} \sin \left(\left(t_{n-j}-s\right) \Omega\right) g\left(X_{s}^{1}\right) d s \\
& +\frac{1}{2} h^{2} \Psi g_{n-j-1}-\frac{1}{2} h^{2} \Psi g\left(\Phi X_{t_{n-j-1}}^{1}\right) .
\end{aligned}
$$

Using Lemma 1 from [4], we get

$$
\begin{aligned}
e_{n-j-1}= & -\frac{1}{2} h^{2}\left(\operatorname{sinc}^{2}\left(\frac{h \Omega}{2}\right)-\Psi\right) g\left(\Phi X_{t_{n-j-1}}^{1}\right) \\
& -h^{3} z_{n-j-1}+\frac{1}{2} h^{2} \Psi g_{n-j-1}-\frac{1}{2} h^{2} \Psi g\left(\Phi X_{t_{n-j-1}}^{1}\right)
\end{aligned}
$$

with $\left\|z_{n-j-1}\right\| \leq C$ and $\left\|h \Omega z_{n-j-1}\right\| \leq C$. It follows, see also Lemma 5 from [4], that

$$
\begin{aligned}
\left\|\sum_{j=0}^{n-1} \Omega \cos (j h \Omega) e_{n-j-1}\right\| \leq & \left\|\frac{1}{2} v_{n-1}\right\|+\left\|h^{2} \sum_{j=0}^{n-1} h \Omega \cos (j h \Omega) z_{n-j-1}\right\| \\
& +\left\|\frac{1}{2} h \sum_{j=0}^{n-1} h \Omega \cos (j h \Omega) \Psi\left(g_{n-j-1}-g\left(\Phi X_{t_{n-j-1}}^{1}\right)\right)\right\|,
\end{aligned}
$$

where $v_{n-1}$ is defined as

$$
v_{n-1}=\frac{1}{2} h \sum_{j=0}^{n-1} h \Omega \cos (j h \Omega)\left(\operatorname{sinc}^{2}\left(\frac{h \Omega}{2}\right)-\Psi\right) g\left(\Phi X_{t_{n-j-1}}^{1}\right)
$$

and can be written as (see Lemma 5 from [4])

$$
v_{n-1}=E_{n-1}^{\prime}(h \Omega) g\left(\Phi X_{0}^{1}\right)+\sum_{j=0}^{n-2} E_{j}^{\prime}(h \Omega)\left(g\left(\Phi X_{t_{n-j-1}}^{1}\right)-g\left(\Phi X_{t_{n-j-2}}^{1}\right)\right),
$$

where

$$
E_{j}^{\prime}(\xi):=\frac{-\xi}{2 \sin \left(\frac{\xi}{2}\right)}\left(\operatorname{sinc}^{2}\left(\frac{\xi}{2}\right)-\psi(\xi)\right)\left(\sin \left(j \xi+\frac{\xi}{2}\right)-\sin \left(\frac{\xi}{2}\right)\right)
$$


Due to (9) and Lemma 5 from [4], $E_{j}^{\prime}$ are bounded and so is $v_{n-1}$. It thus follows that

$$
\begin{aligned}
& \left\|\sum_{j=0}^{n-1} \Omega \cos (j h \Omega) e_{n-j-1}\right\| \leq C h+C h^{2} n \\
& \quad+\left\|\frac{1}{2} h \sum_{j=0}^{n-1} h \Omega \cos (j h \Omega) \Psi\left(g_{n-j-1}-g\left(\Phi X_{t_{n-j-1}}^{1}\right)\right)\right\| .
\end{aligned}
$$

Using the bounds (5) and (9) for the filter functions together with the mean-square error bounds in the position, we obtain

$$
\mathbb{E}\left[\left\|\sum_{j=0}^{n-1} \Omega \cos (j h \Omega) e_{n-j-1}\right\|\right] \leq C h
$$

Now, we have to estimate $\left\|\sum_{j=0}^{n-1} \sin (j h \Omega) e_{n-j-1}^{\prime}\right\|$. To do this, we first rewrite $e_{n-j-1}^{\prime}$ as

$$
\begin{aligned}
e_{n-j-1}^{\prime}= & \frac{h}{2} \Psi_{1} g_{n-j}+\frac{h}{2} \Psi_{0} g_{n-j-1}-\int_{t_{n-j-1}}^{t_{n-j}} \cos \left(\left(t_{n-j}-s\right) \Omega\right) g\left(X_{s}^{1}\right) d s \\
= & \frac{h}{2} \Psi_{1} g_{n-j}+\frac{h}{2} \Psi_{0} g_{n-j-1}-\frac{h}{2} \Psi_{1} g\left(\Phi X_{t_{n-j}}^{1}\right)-\frac{h}{2} \Psi_{0} g\left(\Phi X_{t_{n-j-1}}^{1}\right) \\
& -\int_{t_{n-j-1}}^{t_{n-j}} \cos \left(\left(t_{n-j}-s\right) \Omega\right) g\left(X_{s}^{1}\right) d s+\frac{h}{2} \Psi_{1} g\left(\Phi X_{t_{n-j}}^{1}\right)+\frac{h}{2} \Psi_{0} g\left(\Phi X_{t_{n-j-1}}^{1}\right) \\
= & \frac{h}{2} \Psi_{1} g_{n-j}+\frac{h}{2} \Psi_{0} g_{n-j-1}-\frac{h}{2} \Psi_{1} g\left(\Phi X_{t_{n-j}}^{1}\right)-\frac{h}{2} \Psi_{0} g\left(\Phi X_{t_{n-j-1}}^{1}\right) \\
& -\frac{1}{2} h\left(\operatorname{sinc}(h \Omega)-\Psi_{0}\right) g\left(\Phi X_{t_{n-j-1}}^{1}\right)-\frac{1}{2} h\left(\operatorname{sinc}(h \Omega)-\Psi_{1}\right) g\left(\Phi X_{t_{n-j}}^{1}\right) \\
& -\frac{1}{2} h \int_{0}^{1} \cos ((h-h s) \Omega)\left(g\left(X_{t_{n-j-1}+h s}^{1}\right)-g\left(\Phi X_{t_{n-j-1}}^{1}\right)\right) d s \\
& -\frac{1}{2} h \int_{0}^{1} \cos ((h-h s) \Omega)\left(g\left(X_{t_{n-j-1}+h s}^{1}\right)-g\left(\Phi X_{t_{n-j}}^{1}\right)\right) d s
\end{aligned}
$$

using Lemma 2 from [4]. The triangle inequality gives us

$$
\mathbb{E}\left[\left\|\sum_{j=0}^{n-1} \sin (j h \Omega) e_{n-j-1}^{\prime}\right\|\right] \leq \mathbb{E}\left[\left\|\sum_{j=0}^{n-1} \sin (j h \Omega) \frac{h}{2} \Psi_{1}\left(g_{n-j}-g\left(\Phi X_{t_{n-j}}^{1}\right)\right)\right\|\right]
$$




$$
\begin{aligned}
& +\mathbb{E}\left[\| \sum_{j=0}^{n-1} \sin (j h \Omega) \frac{h}{2} \Psi_{0}\left(g_{n-j-1}-g\left(\Phi X_{t_{n-j-1}}^{1}\right) \|\right]\right. \\
& +\mathbb{E}\left[\left\|\sum_{j=0}^{n-1} \sin (j h \Omega) \cdot d_{n-j-1}^{\prime}\right\|\right],
\end{aligned}
$$

where $d_{n-j-1}^{\prime}$ is defined as

$$
\begin{aligned}
d_{n-j-1}^{\prime}= & \frac{1}{2} h\left(\operatorname{sinc}(h \Omega)-\Psi_{0}\right) g\left(\Phi X_{t_{n-j-1}}^{1}\right)+\frac{1}{2} h\left(\operatorname{sinc}(h \Omega)-\Psi_{1}\right) g\left(\Phi X_{t_{n-j}}^{1}\right) \\
& +\frac{1}{2} h \int_{0}^{1} \cos ((h-h s) \Omega)\left(g\left(X_{t_{n-j-1}+h s}^{1}\right)-g\left(\Phi X_{t_{n-j-1}}^{1}\right)\right) d s \\
& +\frac{1}{2} h \int_{0}^{1} \cos ((h-h s) \Omega)\left(g\left(X_{t_{n-j-1}+h s}^{1}\right)-g\left(\Phi X_{t_{n-j}}^{1}\right)\right) d s .
\end{aligned}
$$

Replacing $\cos (j h \Omega)$ by $\sin (j h \Omega)$ in Lemma 6 from [4] (with a shift in the indices) permits us to bound the terms containing the factors $\operatorname{sinc}(h \Omega)-\Psi_{i}$ for $i=0,1$. For the terms containing the integrals, we use the mean-value theorem and finally, an application of Theorem 2 gives us the desired bound

$$
\mathbb{E}\left[\left\|\sum_{j=0}^{n-1} \sin (j h \Omega) e_{n-j-1}^{\prime}\right\|\right] \leq C h .
$$

We thus finally obtain the estimate

$$
\mathbb{E}\left[\left\|\Omega\left(r_{1}-\hat{r}_{1}\right)\right\|\right] \leq C h .
$$

Theorem 4 Under the assumptions of Theorem 2, the numerical solution (3) of the stochastic oscillator (2) with a smooth gradient nonlinearity $g(x)=-\nabla U(x)$ satisfies

$$
\begin{aligned}
& \mathbb{E}\left[\frac{1}{2}\left(\left\|X_{n}^{2}\right\|^{2}+\left\|\Omega X_{n}^{1}\right\|^{2}\right)+U\left(X_{n}^{1}\right)\right] \\
& \quad=\frac{1}{2}\left(\left\|y_{0}\right\|^{2}+\left\|\Omega x_{0}\right\|^{2}\right)+U\left(x_{0}\right)+\frac{\operatorname{Tr}\left(B B^{T}\right)}{2} t_{n}+\mathcal{O}(h),
\end{aligned}
$$

where $t_{n}=n h \leq T$. The constant symbolised by the $\mathcal{O}$-notation only depends on $T$, $K, M_{1}, \ldots, M_{4},\|g\|,\left\|g^{\prime}\right\|$ and $\left\|g^{\prime \prime}\right\|$, but is independent of $\varepsilon, h$ and $n$ with $n h \leq T$.

Remark 5 One can show that the energy of the numerical solution (3) has exactly the same growth rate as the exact solution of (1) in the case where $g(x) \equiv 0$. The proof is an adaptation of the proof of Theorem 2.2 in [1]. 
Proof Instead of the expected value of the energy

$$
\mathbb{E}\left[\frac{1}{2}\left\|\Omega X_{n}^{1}\right\|^{2}+\frac{1}{2}\left\|X_{n}^{2}\right\|^{2}+U\left(X_{n}^{1}\right)\right]
$$

we will calculate and estimate the expression

$$
\begin{aligned}
\frac{1}{2} \mathbb{E}[ & \left.\left\|\Omega X_{n}^{1}\right\|^{2}-\left\|\Omega X_{n h}^{1}\right\|^{2}+\left\|X_{n}^{2}\right\|^{2}-\left\|X_{n h}^{2}\right\|^{2}\right]+\mathbb{E}\left[U\left(X_{n}^{1}\right)-U\left(X_{n h}^{1}\right)\right] \\
+ & \mathbb{E}\left[\frac{1}{2}\left\|\Omega X_{n h}^{1}\right\|^{2}+\frac{1}{2}\left\|X_{n h}^{2}\right\|^{2}+U\left(X_{n h}^{1}\right)\right] .
\end{aligned}
$$

Since the last term is the expected value of the total energy along the exact solution of our problem, it is thus equal to the initial energy plus the drift:

$\mathbb{E}\left[\frac{1}{2}\left\|\Omega X_{n h}^{1}\right\|^{2}+\frac{1}{2}\left\|X_{n h}^{2}\right\|^{2}+U\left(X_{n h}^{1}\right)\right]=\frac{1}{2}\left\|\Omega x_{0}\right\|^{2}+\frac{1}{2}\left\|y_{0}\right\|^{2}+U\left(x_{0}\right)+\frac{\operatorname{Tr}\left(B B^{T}\right)}{2} n h$.

For the central term, we use the mean-value theorem and Theorem 2 to obtain

$$
\mathbb{E}\left[U\left(X_{n}^{1}\right)-U\left(X_{n h}^{1}\right)\right]=\nabla U(\zeta)^{T} \mathbb{E}\left[X_{n}^{1}-X_{n h}^{1}\right]=\mathcal{O}(h)
$$

Finally, using the notations of Theorem 2, we obtain for the first term:

$$
\begin{aligned}
& \mathbb{E}\left[\left\|\Omega X_{n}^{1}\right\|^{2}-\left\|\Omega X_{n h}^{1}\right\|^{2}+\left\|X_{n}^{2}\right\|^{2}-\left\|X_{n h}^{2}\right\|^{2}\right]=\mathbb{E}\left[\left\|\Omega q_{1}\right\|^{2}+\left\|q_{2}\right\|^{2}-\left\|\Omega \hat{q}_{1}\right\|^{2}\right. \\
& \left.\quad-\left\|\hat{q}_{2}\right\|^{2}\right]+2\left(\Omega p_{1}\right)^{T} \mathbb{E}\left[\Omega\left(r_{1}-\hat{r}_{1}\right)\right]+2 p_{2}^{T} \mathbb{E}\left[r_{2}-\hat{r}_{2}\right]+\mathbb{E}\left[\left\|\Omega r_{1}\right\|^{2}-\left\|\Omega \hat{r}_{1}\right\|^{2}\right] \\
& \quad+\mathbb{E}\left[\left\|r_{2}\right\|^{2}-\left\|\hat{r}_{2}\right\|^{2}\right]+2 \mathbb{E}\left[\left(\Omega q_{1}\right)^{T}\left(\Omega\left(r_{1}-\hat{r}_{1}\right)\right)\right]+2 \mathbb{E}\left[q_{2}^{T}\left(r_{2}-\hat{r}_{2}\right)\right] .
\end{aligned}
$$

We will now estimate each of the above terms. Let us begin with

$$
\begin{aligned}
\mathbb{E}\left[\left\|\Omega q_{1}\right\|^{2}+\left\|q_{2}\right\|^{2}\right]= & \mathbb{E}\left[\sum _ { j = 0 } ^ { n - 1 } \Delta W _ { n - j - 1 } ^ { T } B ^ { T } \left(\sin ^{2}(h \Omega) \cos ^{2}(j h \Omega)\right.\right. \\
& \left.+\sin ^{2}(h \Omega) \sin ^{2}(j h \Omega)\right) B \Delta W_{n-j-1} \\
& +\Delta W_{n-j-1}^{T} B^{T}\left(\cos ^{2}(h \Omega) \sin ^{2}(j h \Omega)\right. \\
& \left.\left.+\cos ^{2}(h \Omega) \cos ^{2}(j h \Omega)\right) B \Delta W_{n-j-1}\right] \\
= & \mathbb{E}\left[n \Delta W_{n-j-1}^{T} B^{T} B \Delta W_{n-j-1}\right]=\operatorname{Tr}\left(B B^{T}\right) n h .
\end{aligned}
$$

Analogously we find $\mathbb{E}\left[\left\|\Omega \hat{q}_{1}\right\|^{2}+\left\|\hat{q}_{2}\right\|^{2}\right]=\operatorname{Tr}\left(B B^{T}\right) n h$ and thus

$$
\mathbb{E}\left[\left\|\Omega q_{1}\right\|^{2}+\left\|q_{2}\right\|^{2}-\left\|\Omega \hat{q}_{1}\right\|^{2}-\left\|\hat{q}_{2}\right\|^{2}\right]=0 .
$$


Next, using Lemma 3 and Theorem 1 we get

$$
2\left(\Omega p_{1}\right)^{T} \mathbb{E}\left[\Omega\left(r_{1}-\hat{r}_{1}\right)\right]+2 p_{2}^{T} \mathbb{E}\left[r_{2}-\hat{r}_{2}\right]=\mathcal{O}(h) .
$$

For the following term, the Cauchy-Schwarz inequality together with Lemma 3 and Theorem 1 give us the bounds

$$
\begin{aligned}
\mathbb{E}\left[\left\|\Omega r_{1}\right\|^{2}-\left\|\Omega \hat{r}_{1}\right\|^{2}\right]+\mathbb{E}\left[\left\|r_{2}\right\|^{2}-\left\|\hat{r}_{2}\right\|^{2}\right]= & \mathbb{E}\left[\left(\Omega\left(r_{1}-\hat{r}_{1}\right)\right)^{T}\left(\Omega\left(r_{1}+\hat{r}_{1}\right)\right)\right] \\
& +\mathbb{E}\left[\left(r_{2}-\hat{r}_{2}\right)^{T}\left(r_{2}+\hat{r}_{2}\right)\right]=\mathcal{O}(h) .
\end{aligned}
$$

Finally, for the last term, we have

$$
2\left(\Omega q_{1}\right)^{T}\left(\Omega\left(r_{1}-\hat{r}_{1}\right)\right)+2 q_{2}^{T}\left(r_{2}-\hat{r}_{2}\right) \leq 2\left\|\Omega q_{1}\right\|\left\|\Omega\left(r_{1}-\hat{r}_{1}\right)\right\|+2\left\|q_{2}\right\|\left\|r_{2}-\hat{r}_{2}\right\|
$$

by the Cauchy-Schwarz inequality. Using Lemma 3 and Theorem 2 we thus obtain

$$
\mathbb{E}\left[2\left(\Omega q_{1}\right)^{T}\left(\Omega\left(r_{1}-\hat{r}_{1}\right)\right)+2 q_{2}^{T}\left(r_{2}-\hat{r}_{2}\right)\right]=\mathcal{O}(h) .
$$

This concludes the proof.

\section{Numerical experiments}

In this final section, we will consider two problems in order to illustrate the robustness of the stochastic trigonometric schemes.

\subsection{The stochastic Fermi-Pasta-Ulam problem}

The deterministic Fermi-Pasta-Ulam (FPU) problem is often used as a model for highly oscillatory problems. For more details on the deterministic case we refer to [5, Chapters I, XIII]. In this section, we will look at a stochastic FPU problem in order to demonstrate the growth rate in the energy and the convergence behaviour of the stochastic trigonometric method.

The deterministic FPU problem describes a chain of $2 \tilde{m}$ mass points, connected with alternating soft nonlinear and stiff linear springs (with angular frequency $\omega:=\frac{1}{\varepsilon} \gg 1$ ). The variables $q_{1}, \ldots, q_{2 \tilde{m}}$ denote the displacements of the mass points, the variables $p_{i}=\dot{q}_{i}$ their velocities. The behaviour of the system is described by a Hamiltonian system wherein the total energy is conserved. After a change of coordinates we obtain the new Hamiltonian function

$$
\begin{aligned}
H(y, x)= & \frac{1}{2} \sum_{i=1}^{2 \tilde{m}} y_{i}^{2}+\frac{\omega^{2}}{2} \sum_{i=1}^{\tilde{m}} x_{\tilde{m}+i}^{2}+\frac{1}{4}\left(\left(x_{1}-x_{\tilde{m}+1}\right)^{4}\right. \\
& \left.+\sum_{i=1}^{\tilde{m}-1}\left(x_{i+1}-x_{\tilde{m}+i+1}-x_{i}-x_{\tilde{m}+i}\right)^{4}+\left(x_{\tilde{m}}+x_{2 \tilde{m}}\right)^{4}\right),
\end{aligned}
$$


where $x_{i}, i=1, \ldots, \tilde{m}$ represents a scaled displacement of the $i$ th stiff spring, $x_{\tilde{m}+i}$ a scaled expansion of the $i$ th stiff spring and $y_{i}$ and $y_{\tilde{m}+i}$ their velocities.

Furthermore, in the deterministic case, another quantity is almost conserved, the oscillatory energy. Let

$$
I_{j}\left(x_{\tilde{m}+j}, y_{\tilde{m}+j}\right)=\frac{1}{2}\left(y_{\tilde{m}+j}^{2}+\omega^{2} x_{\tilde{m}+j}^{2}\right)
$$

denote the energy of the $j$ th stiff spring. As time passes, there is an exchange of energy between the stiff springs, but the total oscillatory energy of our problem

$$
I=\sum_{i=1}^{\tilde{m}} I_{j}
$$

remains almost conserved.

Writing down the Hamiltonian equations, we obtain the following second-order differential equation:

$$
\ddot{x}+\Omega^{2} x=g(x) \text { with } \Omega=\left(\begin{array}{cc}
0 & 0 \\
0 & \omega I
\end{array}\right),
$$

where $I$ is the $\tilde{m} \times \tilde{m}$ identity matrix and the frequency $\omega$ is supposed to be large.

Now we will turn to the stochastic Fermi-Pasta-Ulam problem. By adding a noise term to the equation (14) we get the system

$$
\ddot{X}_{t}+\Omega^{2} X_{t}=g\left(X_{t}\right)+B \dot{W}_{t} .
$$

Since the matrix $\Omega$ is not positive-definite, the convergence of the stochastic trigonometric methods is not obvious. However, setting $\Omega=0$, the expression for the mean-square error in the position in the proof of Proposition 1 reduces to

$$
\begin{aligned}
\mathbb{E}\left[\left\|X_{n}^{1}-X_{n h}^{1}\right\|^{2}\right] & =\sum_{j=0}^{n} \mathbb{E}\left[\left\|\left(d_{j}^{1}+((n-j) h) d_{j}^{2}\right)\right\|^{2}\right] \\
& =\sum_{j=0}^{n} \mathbb{E}\left[\int_{t_{j}}^{t_{j+1}}\left\|\left(t_{j+1}-s-h\right) B\right\|^{2} d s\right] \\
& =\|B\|^{2} \sum_{j=0}^{n} \int_{t_{j}}^{t_{j+1}}\left(t_{j}-s\right)^{2} d s \\
& =\frac{1}{3}\|B\|^{2} \sum_{j=0}^{n} h^{3}
\end{aligned}
$$

since $d_{j}^{2}=0$ and thus we obtain

$$
\left(\mathbb{E}\left[\left\|X_{n}^{1}-X_{n h}^{1}\right\|^{2}\right]\right)^{1 / 2} \leq C h,
$$


for $t_{n}=n h \leq T$. For the mean-square error in the velocity we find analogously

$$
\left(\mathbb{E}\left[\left\|X_{n}^{2}-X_{n h}^{2}\right\|^{2}\right]\right)^{1 / 2} \leq C h
$$

For the expression for the error in the second moment for the position in the proof of Proposition 2 we obtain in the case $\Omega=0$ :

$$
\begin{aligned}
\mid \mathbb{E}[ & \left.\left\|X_{n}^{1}\right\|^{2}\right]-\mathbb{E}\left[\left\|X_{n h}^{1}\right\|^{2}\right] \mid \\
& =\left|\sum_{j=0}^{n-1} \mathbb{E}\left[\left\|(h+j h) B \Delta W_{n-j-1}\right\|^{2}\right]-\sum_{j=0_{t_{n-j-1}}}^{n-1} \int_{t_{n-j}}^{t}\left\|\left(t_{n-j}-s+j h\right) B\right\|^{2} d s\right| \\
& =\left|\sum_{j=0}^{n-1}(h+j h)^{2} h\|B\|^{2}-\sum_{j=0}^{n-1} \int_{t_{n-j-1}}^{t_{n-j}}\left(t_{n-j}-s+j h\right)^{2}\|B\|^{2} d s\right| \\
& =\left|\sum_{j=0}^{n-1}\|B\|^{2}\left(h(h+j h)^{2}+\frac{1}{3}(j h)^{3}-\frac{1}{3}(j h+h)^{3}\right)\right| \\
& =\left|\sum_{j=0}^{n-1}\|B\|^{2}\left(j h^{3}+2 / 3 h^{3}\right)\right| \\
& \leq C h .
\end{aligned}
$$

Analogously we get for the velocity component

$$
\left|\mathbb{E}\left[\left\|X_{n}^{2}\right\|^{2}\right]-\mathbb{E}\left[\left\|X_{n h}^{2}\right\|^{2}\right]\right| \leq C T
$$

Due to the block structure of the matrix $\Omega$ we can split the equation (15) into two equations and write $X_{t}^{1}=\left(X_{t, 1}^{1} X_{t, 2}^{1}\right)^{T}$ and $X_{t}^{2}=\left(X_{t, 1}^{2} X_{t, 2}^{2}\right)^{T}$, where $X_{t, 1}^{1}$, $X_{t, 1}^{2}, X_{t, 2}^{1}, X_{t, 2}^{2} \in \mathbb{R}^{\tilde{m}}$. The slow component of the system will thus be $X_{t, 1}^{1}$ and the fast one $X_{t, 2}^{1}$. Therefore we obtain for the mean-square error in position

$$
\left(\mathbb{E}\left[\left\|X_{n}^{1}-X_{n h}^{1}\right\|^{2}\right]\right)^{1 / 2}=\left(\mathbb{E}\left[\left\|X_{n, 1}^{1}-X_{n h, 1}^{1}\right\|^{2}\right]+\mathbb{E}\left[\left\|X_{n, 2}^{1}-X_{n h, 2}^{1}\right\|^{2}\right]\right)^{1 / 2} \leq C h
$$

and similarly for the velocity component

$$
\left(\mathbb{E}\left[\left\|X_{n}^{2}-X_{n h}^{2}\right\|^{2}\right]\right)^{1 / 2} \leq C T^{1 / 2}
$$

For the errors in the second moment, we get

$$
\left|\mathbb{E}\left[\left\|X_{n}^{1}\right\|^{2}\right]-\mathbb{E}\left[\left\|X_{n h}^{1}\right\|^{2}\right]\right| \leq C h, \quad\left|\mathbb{E}\left[\left\|X_{n}^{2}\right\|^{2}\right]-\mathbb{E}\left[\left\|X_{n h}^{2}\right\|^{2}\right]\right| \leq C T .
$$


For our numerical experiments, we will take $2 \tilde{m}=6$ mass points, a spring constant $\omega=50$, and we will consider

$$
x_{1}(0)=1, \quad y_{1}(0)=1, \quad x_{4}(0)=\omega^{-1}, \quad y_{4}(0)=1
$$

and zero for the remaining initial values. For the stochastic term we choose

$$
B=\left(\begin{array}{llllll}
0 & 1 & 0.5 & 5 & 0 & 0.01
\end{array}\right)^{T}
$$

We finally want to note that all the expected values are computed numerically using sample averages. In order to make these approximations as accurate as necessary, we took enough sample paths in all our numerical experiments.

Figure 1 displays the linear growth rate of the expectation of the energy along the numerical solution of the stochastic trigonometric method (3) with filters given in Example 3 and along the numerical solution given by the Euler-Maruyama scheme. The growth rate given by Theorem 4 is observed and on the contrary, if we solve (15) by the Euler-Maruyama method, the expectation of the total energy of the system grows exponentially.

In Fig. 2 we notice a linear growth of the total oscillatory energy in the stochastic case. As a comparison, we plot the oscillatory energy of the deterministic FPU problem. Both in the deterministic and in the stochastic case, there is an exchange of energy between the stiff springs.

The convergence behaviour of the method is illustrated in Figs. 3 and 4. We calculated $M=20,000$ sample paths for the mean-square convergence and for the convergence in the second moment. For the error in the second moment in the velocity component we chose $\omega=10$ in order to avoid the large number of samples that would be required for $\omega=50$.

Finally, in order to illustrate the fact that our error bounds are independent of the large parameter $\omega$, we chose $\omega=500$ in Fig. 5 and observe the same orders of convergence for the position component as for smaller $\omega$.
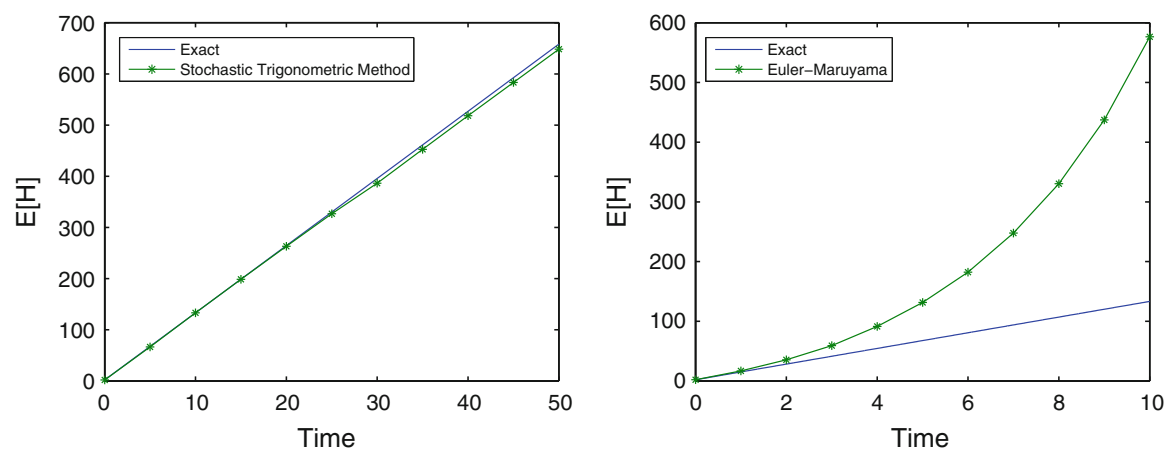

Fig. 1 The stochastic trigonometric method (3) with step size $h=0.1$ and $M=10,000$ sample paths reproduces the linear growth of the energy almost exactly (left picture), while the numerical energy obtained by the Euler-Maruyama method with step size $h=10^{-4}$ and $M=10,000$ sample paths grows exponentially (right picture) 

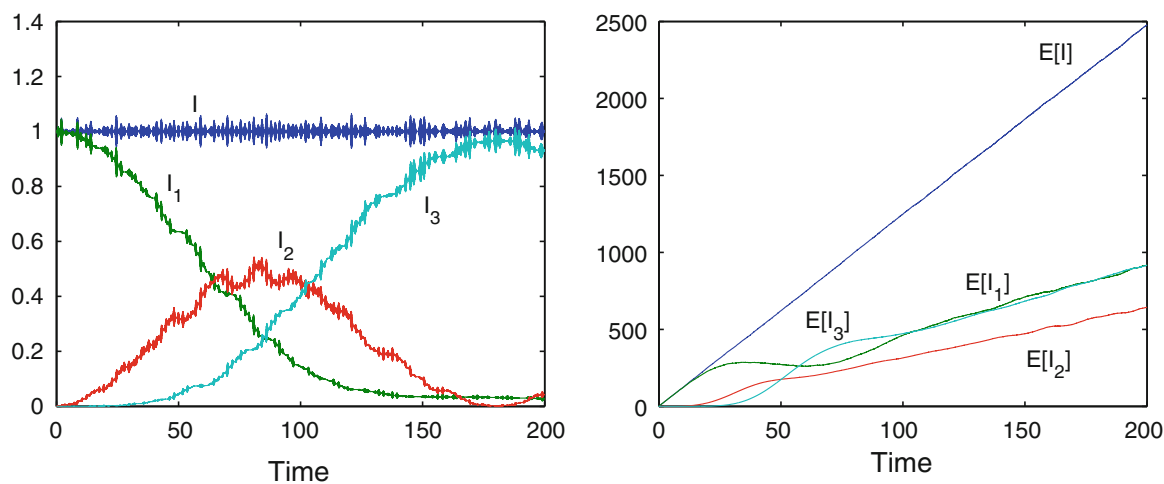

Fig. 2 In the deterministic case the total oscillatory energy is almost conserved, while the stiff springs exchange energy among each other (left picture). In the stochastic case there is also an exchange of energy between the stiff springs, but the expected value of the total oscillatory energy obtained by method (3) with step size $h=0.01$ and $M=10,000$ sample paths grows linearly (right picture)
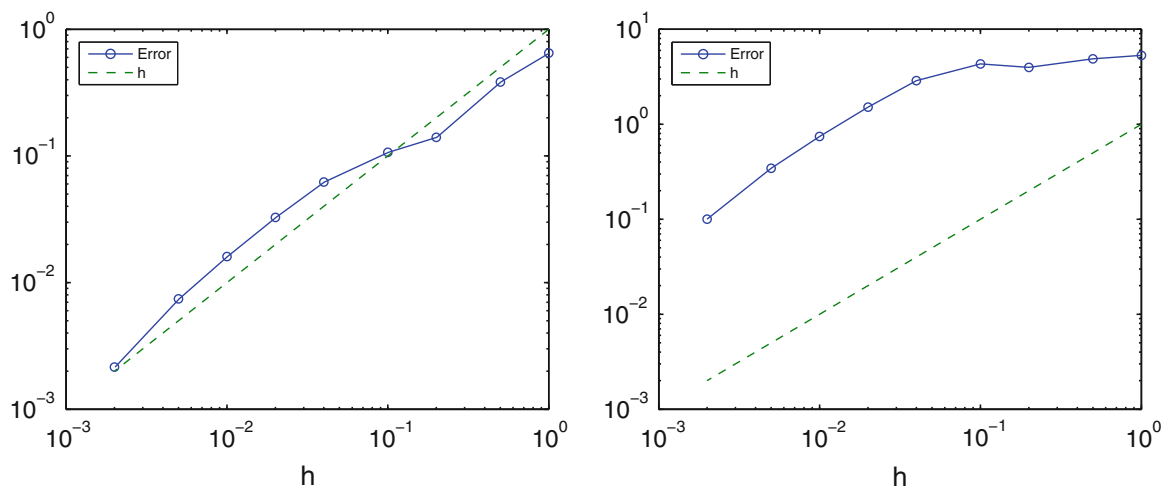

Fig. 3 Mean-square error in the position (left picture) and in the velocity (right picture) for the stochastic FPU problem (15). The dashed lines have slope one
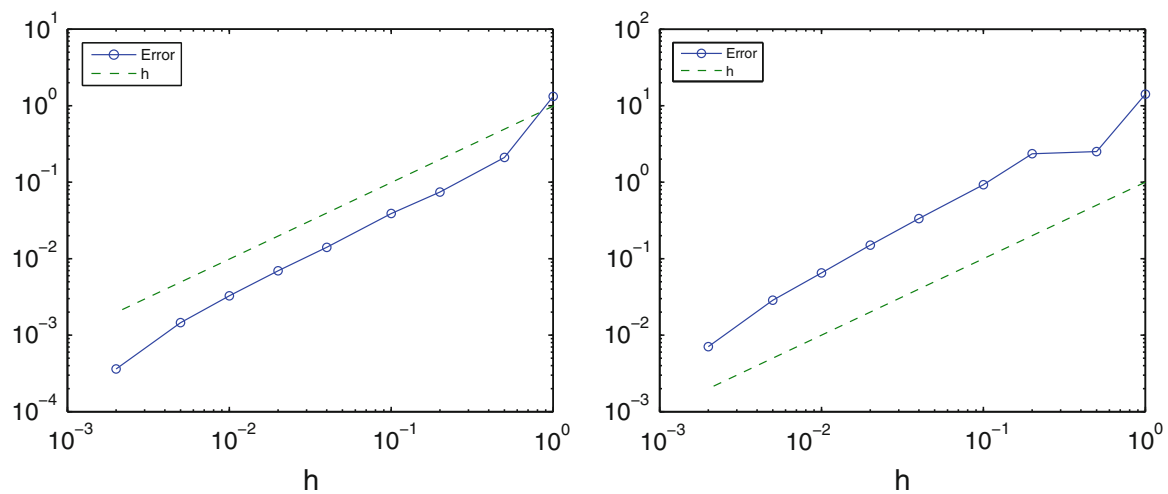

Fig. 4 Error in the second moment for the position (left picture) and for the velocity (right picture) component for the stochastic FPU problem (15). The dashed lines have slope one 

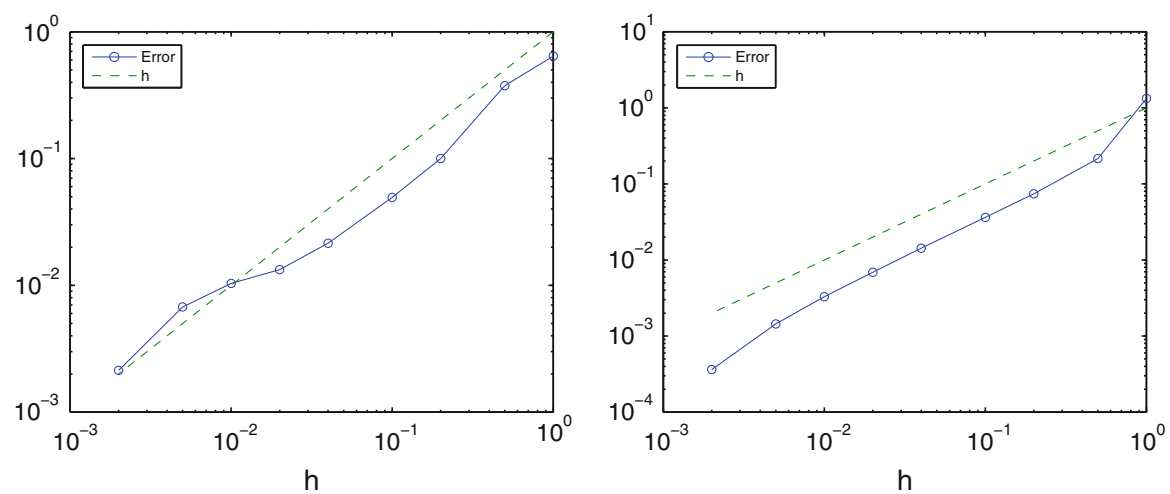

Fig. 5 Mean-square error (left picture) and error in the second moment (right picture) for the position component for the stochastic FPU problem (15) with $\omega=500$. The dashed lines have slope one

\subsection{Semi-discretisation of a semi-linear stochastic wave equation}

As a final example, we consider the pseudo-spectral semi-discretisation (using the eigenfunctions $\left.e_{n}(x)=\sqrt{2 / \pi} \sin (n x)\right)$ of the semi-linear stochastic wave equation from [15]:

$$
u_{t t}(x, t)=\sigma^{2} u_{x x}(x, t)+\left(a_{0}-a_{2}|u(x, t)|_{L^{2}}^{2}\right) u(x, t)+b \xi,
$$

where $(x, t) \in(0, \pi) \times(0, \infty)$ and $\xi$ is white in time and spatially correlated. We impose homogeneous boundary conditions $u(0, t)=u(\pi, t)=0$. This gives us the following system of SDEs for the Fourier coefficients $c_{n}=c_{n}(t)$ :

$$
\ddot{c}_{n}+\left(\sigma^{2} n^{2}-a_{0}\right) c_{n}+a_{2}\left(\sum_{m=1}^{\infty} c_{m}^{2}\right) c_{n}=b_{n} \dot{W}_{n} .
$$

Truncating this system at $N F$ Fourier modes, we end up with a system of the form (1).

To illustrate the excellent long-time behaviour of the stochastic trigonometric scheme (3) with filter functions given by Example 3, we compute the expected energy from Theorem 4, i.e. the trace formula in [15]:

$$
\begin{aligned}
& \mathbb{E}\left[\frac{1}{2} \sum_{n=1}^{N F}\left(v_{n}(t)^{2}+\left(\sigma^{2} n^{2}-a_{0}\right) c_{n}(t)^{2}\right)+\frac{a_{2}}{4}\left(\sum_{n=1}^{N F} c_{n}(t)^{2}\right)^{2}\right] \\
& \quad=\frac{1}{2} \sum_{n=1}^{N F}\left(v_{n}(0)^{2}+\left(\sigma^{2} n^{2}-a_{0}\right) c_{n}(0)^{2}\right)+\frac{a_{2}}{4}\left(\sum_{n=1}^{N F} c_{n}(0)^{2}\right)^{2}+\sum_{n=1}^{N F} b_{n}^{2} \frac{t}{2},
\end{aligned}
$$

where $N F$ is the number of Fourier modes and $v_{n}(t)=\dot{c}_{n}(t)$. Figure 6 shows the expected value of the energy for the following parameters: $N F=1024$ Fouriermodes, $\sigma=1, a_{0}=0.5, a_{2}=0.2, b_{n}=1, M=1000$ samples, $h=0.2$ time step 


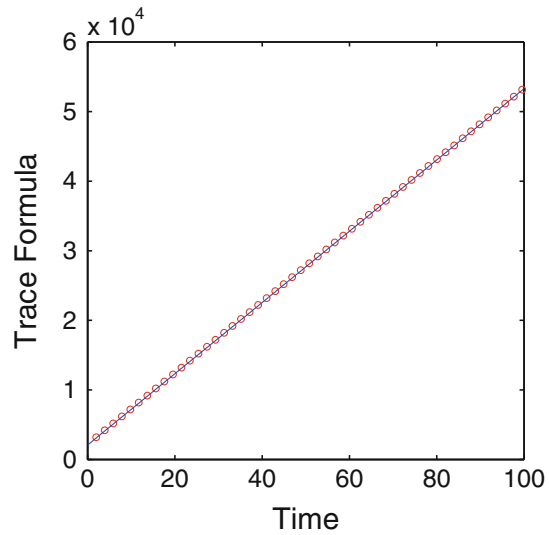

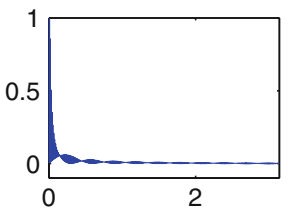

Time $t=0$

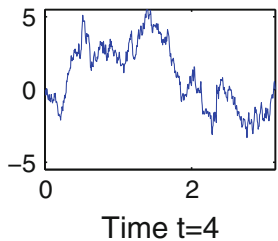

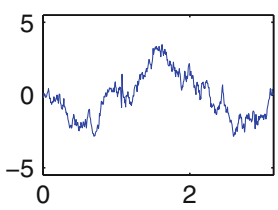

Time $\mathrm{t}=2$

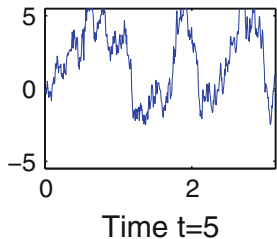

Fig. 6 Linear growth rate in the energy of the stochastic wave equation along the numerical solution given by (3) (left). One sample numerical trajectory (right)

and initial values $c_{n}(0)=0$ and $v_{n}(0)=0$ except for the first 500 Fourier modes, where we set $c_{n}(0)=0.01$. The time interval ranges from $T=0$ to $T=100$. We also display one sample trajectory of the numerical solution to the wave equation. A detailed analysis of the good behaviour of the stochastic trigonometric methods for the time discretisation of the stochastic wave equation will be presented in a forthcoming publication.

Acknowledgments We greatly appreciate the referees' comments and references on an earlier version. We would like to thank Marcus Grote, Luc Guyot, Carlo Marinelli and Lluis Quer-Sardanyons for interesting discussions. This work was partially supported by the Fonds National Suisse, project No. 200021_127130. A large part of this work was carried out when one of the authors (DC) visited the Hausdorff Institute for Mathematics in Bonn. DC would like to thank this institute for its hospitality.

\section{References}

1. Cohen, D.: On the numerical discretisation of stochastic oscillators (2010, Submitted)

2. Cohen, D., Jahnke, T., Lorenz, K., Lubich, C.: Numerical integrators for highly oscillatory Hamiltonian systems: a review. In: Analysis, Modeling and Simulation of Multiscale Problems, pp. 553-576. Springer, Berlin (2006)

3. Deuflhard, P.: A study of extrapolation methods based on multistep schemes without parasitic solutions. Z. Angew. Math. Phys. 30(2), 177-189 (1979)

4. Grimm, V., Hochbruck, M.: Error analysis of exponential integrators for oscillatory second-order differential equations. J. Phys. A 39(19), 5495-5507 (2006)

5. Hairer, E., Lubich, C., Wanner, G.: Geometric numerical integration. Structure-preserving algorithms for ordinary differential equations. Springer Series in Computational Mathematics, vol. 31. Springer, Berlin (2002)

6. Hochbruck, M., Lubich, C., Selhofer, H.: Exponential integrators for large systems of differential equations. SIAM J. Sci. Comput. 19(5), 1552-1574 (1998)

7. Hochbruck, M., Ostermann, A.: Exponential integrators. Acta Numer. 19, 209-286 (2010)

8. Hong, J., Scherer, R., Wang, L.: Predictor-corrector methods for a linear stochastic oscillator with additive noise. Math. Comput. Model. 46(5-6), 738-764 (2007)

9. Jentzen, A., Kloeden, P.E.: Overcoming the order barrier in the numerical approximation of stochastic partial differential equations with additive space-time noise. Proc. R. Soc. Lond. Ser. A Math. Phys. Eng. Sci. 465(2102), 649-667 (2009) 
10. Kloeden, P.E., Lord, G.J., Neuenkirch, A., Shardlow, T.: The exponential integrator scheme for stochastic partial differential equations: pathwise error bounds. J. Comput. Appl. Math. 235(5), 1245-1260 (2011)

11. Kloeden, P.E., Platen, E.: Numerical solution of stochastic differential equations. In: Applications of Mathematics (New York), vol. 23. Springer, Berlin (1992)

12. Leimkuhler, B., Reich, S.: Simulating Hamiltonian dynamics. In: Cambridge Monographs on Applied and Computational Mathematics, vol. 14. Cambridge University Press, Cambridge (2004)

13. Lord, G.J., Rougemont, J.: Topological and $\epsilon$-entropy for large volume limits of discretized parabolic equations. SIAM J. Numer. Anal. 40(4), 1311-1329 (2002)

14. Lord, G.J., Rougemont, J.: A numerical scheme for stochastic PDEs with Gevrey regularity. IMA J. Numer. Anal. 24(4), 587-604 (2004)

15. Schurz, H.: Analysis and discretization of semi-linear stochastic wave equations with cubic nonlinearity and additive space-time noise. Discrete Contin. Dyn. Syst. Ser. S 1(2), 353-363 (2008)

16. Strømmen Melbø, A.H., Higham, D.J.: Numerical simulation of a linear stochastic oscillator with additive noise. Appl. Numer. Math. 51(1), 89-99 (2004)

17. Tao, M., Owhadi, H., Marsden, J.E.: Structure preserving stochastic impulse methods for stiff langevin systems with a uniform global error of order 1 or $1 / 2$ on position. arXiv:1006.4657v1 (2010)

18. Tocino, A.: On preserving long-time features of a linear stochastic oscillator. BIT 47(1), 189-196(2007) 